\title{
GSI-Based, Continuously Cycled, Dual-Resolution Hybrid Ensemble-Variational Data Assimilation System for HWRF: System Description and Experiments with Edouard (2014)
}

\author{
Xu Lu AND Xuguang WANG \\ School of Meteorology, University of Oklahoma, Norman, Oklahoma \\ MingJing TONG AND ViJAY TALLAPRAGADA \\ Environmental Modeling Center, NOAA/National Centers for Environmental Prediction, College Park, Maryland
}

(Manuscript received 22 March 2017, in final form 10 October 2017)

\begin{abstract}
A Gridpoint Statistical Interpolation analysis system (GSI)-based, continuously cycled, dual-resolution hybrid ensemble Kalman filter-variational (EnKF-Var) data assimilation (DA) system is developed for the Hurricane Weather Research and Forecasting (HWRF) Model. In this system, a directed moving nest strategy is developed to solve the issue of nonoverlapped domains for cycled ensemble DA. Additionally, both dual-resolution and four-dimensional ensemble-variational (4DEnVar) capabilities are implemented. Vortex modification (VM) and relocation (VR) are used in addition to hybrid DA. Several scientific questions are addressed using the new system for Hurricane Edouard (2014). It is found that dual-resolution hybrid DA improves the analyzed storm structure and short-term maximum wind speed (Vmax) and minimum sea level pressure (MSLP) forecasts compared to coarser, single-resolution hybrid DA, but track and radius of maximum wind (RMW) forecasts do not improve. Additionally, applying VR and VM on the control background before DA improves the analyzed storm, overall track, RMW, MSLP, and Vmax forecasts. Further applying VR on the ensemble background improves the analyzed storm and forecast biases for MSLP and Vmax. Also, using 4DEnVar to assimilate tail Doppler radar (TDR) data improves the analyzed storm and short-term MSLP and Vmax forecasts compared to three-dimensional ensemble-variational (3DEnVar) although 4DEnVar slightly degrades the track forecast. Finally, the new system improves overall RMW, MSLP, and Vmax forecasts upon the operational HWRF, while no improvement on track is found. The intensity forecast improvement during the intensifying period is due to the better analyzed structures for an intensifying storm.
\end{abstract}

\section{Introduction}

An accurate estimation of the background error covariance is required to effectively assimilate observations to initialize numerical predictions of tropical cyclones (TCs). Early studies have demonstrated the promise of using ensemble covariances for TC data assimilation (DA). In these studies, the ensemble covariances were adopted either through the use of the ensemble Kalman filter (EnKF; e.g., Zhang et al. 2009; Torn 2010; Aksoy et al. 2012; Weng and Zhang 2012; Poterjoy et al. 2014; Poterjoy and Zhang 2014) or the ensemble-variational hybrid (EnVar) method (e.g., Wang 2011; Li et al. 2012; Schwartz et al. 2013, 2015; Wang and Lei 2014; Li et al. 2015; Lu et al. 2017). These studies have shown that using

Corresponding author: Xuguang Wang, xuguang.wang@ou.edu such covariances improved track and intensity forecasts due to superior analyses of the TC environment (Wang 2011; Poterjoy and Zhang 2014; Zhang and Weng 2015), TC vortex, and inner-core structures (Li et al. 2012; Weng and Zhang 2012; Lu et al. 2017).

Starting from 2013, the Gridpoint Statistical Interpolation analysis system (GSI)-based hybrid DA method was implemented for the operational Hurricane Weather Research and Forecasting (HWRF) Model following the successful implementation of the hybrid DA system for the operational Global Forecast System (GFS) (e.g., Wang et al. 2013; Wang and Lei 2014; Kleist and Ide 2015a,b). In operational HWRF DA, either the GFS ensemble forecast or an HWRF ensemble initialized from GFS analyses has been used to provide the flowdependent background error covariances (M. Tong 2016, personal communication). However, these ensembles are 
inherently inconsistent with the errors in the HWRF background forecast since the GFS analyses and forecasts are produced from a different model with a different resolution and assimilate different sets of observations. Therefore, these ensembles are not able to accurately estimate the error covariances associated with the higherresolution HWRF TC background forecast initialized from its own DA. We hypothesize that this inconsistency significantly degrades HWRF forecasts. ${ }^{1}$

To investigate the hypotheses, a GSI-based hybrid EnVar DA system that uses the self-consistent HWRF EnKF ensemble was further developed for HWRF (Lu et al. 2017). As an initial effort, the system used a fixed, single nest domain and covered only a small period of the TC life that was surveyed by the tail Doppler radar (TDR) observations. Lu et al. (2017) found that the corrections of the wind and mass fields by this hybrid DA system were dynamically and thermodynamically consistent. The self-consistent HWRF EnKF ensemble was superior to the GFS ensemble when ingested by the hybrid system for improving both the analyzed structures and forecasts of the TCs. In addition, the selfconsistent hybrid system for HWRF exhibited the most benefits of assimilating airborne radar data.

In this study, the HWRF hybrid DA system is further developed to address both the scientific and technical challenges to perform end-to-end, continuous DA and forecast cycles for the entire life of a TC, and assimilating all operational observations in addition to TDR. Triply nested domains with movable inner nests are adopted during the continuous DA and forecast cycles. A remaining challenge is that the locations of the inner nests will diverge in the background ensemble if the stormcenter-following moving nests are adopted. The current ensemble DA system developed based on GSI is not able to provide state updates in areas where the background ensemble nests are not collocated. A directed moving nest strategy is developed in this study to address this issue.

The positive impact of using a high-resolution NWP model in TC prediction has been found in numerous studies over the past decade (e.g., Davis and Bosart 2002; Zhu et al. 2004; Davis et al. 2010; Chen et al. 2011; Zhang et al. 2011; Xue et al. 2013). Early studies suggested that model grid that is at least convection allowing is required to resolve the convective-scale features such as the moist convection and eyewall's dynamics (e.g., Houze et al. 2007; Zhang et al. 2011). While the operational HWRF has

\footnotetext{
${ }^{1}$ Starting in 2017, the operational HWRF will have the capability for the self-cycled background error covariances using a new system based on that presented here (J. Sippel 2017, personal communication).
}

become convection allowing (Tallapragada et al. 2014), running all ensemble members at convection-allowing resolution for ensemble DA still poses challenges due to limited computing resources available at the operational centers.

Some recent studies (e.g., Li 2015; Schwartz et al. 2015) have implemented a "dual resolution" hybrid DA method for hurricane analysis and forecast. Using the dual-resolution method, the control background forecast is run at higher resolution than the ensemble members that form the background error covariances. This approach both resolves the convective-scale structures and reduces the computational cost. Schwartz et al. (2015) showed that dual-resolution hybrid DA can efficiently reduce the computational cost with minimal degradation relative to full-resolution ensemble DA. However, Schwartz et al. (2015) only explored the impact of dualresolution configuration in a coarse-resolution scenario where the control background and analysis were still at convection-parameterizing resolution. Later, Schwartz (2016) applied the dual-resolution DA method to precipitation forecasts over the conterminous United States (CONUS). In their dual-resolution DA settings, a control background used 4-km convection-allowing horizontal grid spacing while the ensembles used $20-\mathrm{km}$ convectionparameterizing horizontal grid spacing. The dualresolution DA was found to improve the precipitation forecasts for the first $12 \mathrm{~h}$ upon the single-resolution DA where both control background and ensembles are at 20-km convection-parameterizing horizontal grid spacing. The dual-resolution configuration has not been examined for hurricane prediction, and this study is the first to extend the capability with the newly developed HWRF hybrid DA system. In particular, the control analysis and background is run at the convection-allowing 3-km horizontal grid spacing whereas the ensemble background is run at the 9-km convection-parameterizing horizontal grid spacing. Using an experiment where all control and the ensemble are run at the 9-km horizontal grid spacing reveals the impact of introducing a convection-allowing control analysis and background for hurricane prediction.

While satellites provide the majority of observations over the open ocean, effective utilization of cloudcontaminated satellite observation in DA still remains a challenge (e.g., Bauer et al. 2006, 2011; Yang et al. 2016; Zhang et al. 2016). Although various field campaigns provide unprecedented and valuable inner-core observations of TCs over the open water [e.g., Hurricane Intensity Forecasting Experiment (IFEX) field campaign (Rogers et al. 2013b); Sensing Hazards with Operational Unmanned Technology (SHOUT) field campaign (Coffey et al. 2015); Tropical Cyclone Intensity Experiment (TCI) field campaign (Doyle et al. 2017)], it is unusual to have routine sampling of the inner core of a TC 
for its entire lifetime. To partly solve this issue, early studies used bogus vortex methods to initialize vortex position and structure based on limited vortex information (e.g., Kurihara et al. 1990, 1993, 1995; Lord 1991; Thu and Krishnamurti 1992; Bender et al. 1993; Pu and Braun 2001; Liu et al. 2000, 2006; Tallapragada et al. 2014). In the operational HWRF system, a similar vortex initialization (VI) procedure is used and primarily contains two components: 1) storm location correction and 2) storm size and intensity correction (Liu et al. 2000, 2006). Hereafter, in this manuscript, the vortex relocation (VR) refers to only the location correction and the vortex modification (VM) refers to the storm size and intensity correction. In the meantime, recent studies showed that advanced DA methods can effectively extrapolate observation information without relying on the typically used VI when the inner-core observations are available (Torn and Hakim 2009; Xiao et al. 2009; Zhang et al. 2009, 2011; Li et al. 2012; Weng and Zhang 2012; Aksoy et al. 2013; Schwartz et al. 2013; Lu et al. 2017). Schwartz et al. (2013) showed that when "bogus" inner-core observations are assimilated efficiently and continuously in a convection-parameterizing horizontal grid spacing $(45 \mathrm{~km})$ with a cycled, hybrid DA system, VR does not improve the hybrid DA for typhoon track forecasts. This study explores the extent that VI is still necessary in a fully cycled convection-allowing DA system where innercore observations are only partially available and how the VI can be integrated with the DA.

In the operational HWRF, observations are assimilated using fixed error covariances that do not evolve over the 6-h DA window. When storms are rapidly changing, such as going through rapid intensification (RI) and eyewall replacement, DA methods that account for the temporal evolution of the error covariances within the 6-h window are likely needed. Additionally, while the analysis is valid at the center of the 6-h DA window, the inner-core observations are usually not valid at the analysis time but rather distributed over the 6-h window depending on the aircraft flight times. In such cases, using a four-dimensional error covariance will properly update the state at the analysis time. Wang and Lei (2014) illustrated in the GFS model that the GSI-based 4DEnVar can effectively resolve temporal evolution of the error covariances for hurricane analysis. However, impact of $4 \mathrm{DEnVar}$ in the context of convection-allowing inner-core DA has not been explored. Therefore, a 4DEnVar capability is further implemented to the HWRF hybrid system to address this question.

This manuscript first describes the newly extended GSI-based, continuously cycled, dual-resolution hybrid ensemble-variational DA system for HWRF with various capabilities, including the 3DEnVar, 4DEnVar, dualresolution, and the directed moving nest strategy (section 2). Using the newly extended hybrid system, section 3 describes experiments designed to assess the impact of 1) introducing a high-resolution control analysis and forecast through dual-resolution DA, 2) integrating VI with DA, and 3) accounting for the temporal evolution of the error covariance with 4DEnVar. Section 4 discusses the results associated with these experiments. In addition, as part of evaluating and understanding the performance of the newly developed system, section 4 also explores the potential of the newly developed HWRF hybrid DA system to further improve intensity forecast by diagnosing the analyzed storm structure and comparing the intensity forecast with the operational HWRF. Section 5 concludes the paper. As a first step of evaluating the newly developed system and to address the aforementioned scientific and technical challenges, the cycling DA and forecast experiments were conducted for Hurricane Edouard (2014). Systematic experiments are planned in the future.

\section{Methodologies and system description}

\section{a. Overview of the GSI-based, continuously cycled, dual-resolution hybrid ensemble-variational (EnVar) DA system for HWRF}

Following the early development (Lu et al. 2017), the GSI-based hybrid EnKF-Var DA system for HWRF is further extended to integrate with VI and to include the continuously cycling, dual-resolution 3DEnVar and 4DEnVar capabilities. Figure 1 shows an example of the flowchart of the newly developed system, and a detailed description follows below.

For the first cycle of a storm, a 40-member HWRF ensemble is initialized by the ensemble analyses from the National Centers for Environmental Prediction (NCEP) operational GFS hybrid DA system (Wang et al. 2013). In the meantime, a single deterministic HWRF spinup forecast is initialized by the control analysis from the same GFS system. Using the Tropical Cyclone Vitals (TCVital) database and following Liu et al. (2000, 2006), vortices in the 6-h ensemble forecast are relocated, while vortices in the control forecast valid at 3,6 , and $9 \mathrm{~h}$ are both relocated and modified before meeting the requirement of first guess at appropriate time (FGAT; ${ }^{2}$ see Trahan and Sparling 2012). The modified control forecast is then used as the control background for hybrid DA on 3- and 9-km grids, while the relocated ensemble forecast

\footnotetext{
${ }^{2}$ FGAT interpolates the background forecast to the observation time so that the observation priors in the innovation correspond to the "appropriate" observation time. To perform the FGAT interpolation, background forecasts at 3,6 , and $9 \mathrm{~h}$ are used for a 6-h DA time window centered at $6 \mathrm{~h}$.
} 


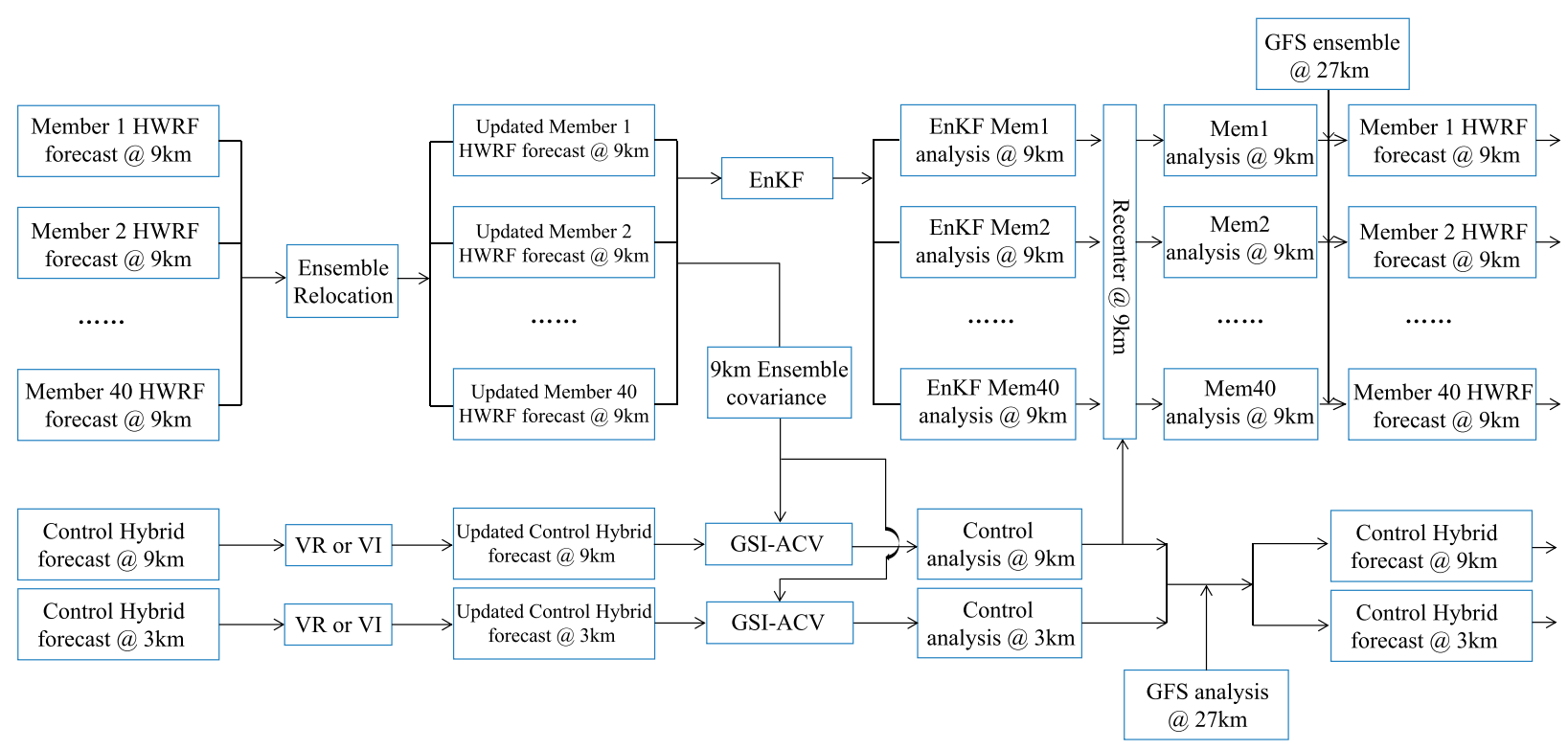

FIG. 1. Flowchart of GSI-based EnKF-variational hybrid data assimilation system for HWRF [adapted from Wang et al. (2013)].

is used as the background ensemble for the EnKF on the 9-km grid and for hybrid DA on both the 3- and 9-km grids. Further details regarding VR and VM procedures are discussed in section 2e. During the continuously cycled ensemble and control background forecasts, a newly developed directed moving nest strategy is adopted. A detailed description of the directed moving nest strategy will be discussed in section $2 \mathrm{~d}$.

At the DA and state update stage, four steps are involved. First, the 40-member relocated HWRF background forecast is updated by the EnKF to produce a 40-member analysis on the 9-km domain. Next, the control analysis on the 3- and 9-km domains is produced with GSI hybrid DA using the 40-member relocated HWRF ensemble and the augmented control vector (ACV) method in GSI variational minimization (GSI-ACV; Wang et al. 2013). Since the assimilation window is $6 \mathrm{~h}$, FGAT is applied for the hybrid DA. Then the EnKF analysis on the 9-km domain is then recentered around the 9-km hybrid analysis (i.e., the EnKF mean is replaced with the hybrid analysis). Finally, the 27-km HWRF outermost domain is replaced by the GFS control and ensemble analyses without undergoing DA. The DA is not performed on the outermost domain following the operational HWRF, a configuration chosen because of limited benefits from using a regional analysis instead of the global analysis for the large-scale TC environment (Tallapragada et al. 2014).

After the analyses are produced from the above four steps, the background forecasts are produced to prepare for the next DA cycle. In particular, the hybrid analysis is used to initialize a 9-h short-term deterministic forecast on the 27/9/3-km grid, and a 6-h 40-member ensemble background forecasts on the $27 / 9-\mathrm{km}$ grids is initialized from the recentered HWRF EnKF analysis. The same directed moving nest strategy, VR, and VM procedures as applied for the spinup background forecasts are then adopted before applying the next DA steps. This cycling of DA and forecasts continues through the end of the storm.

Finally, to evaluate various DA methods, a single deterministic 120 -h free forecast is initialized by the hybrid analysis for each DA cycle. This forecast is independent of the 9-h control forecast that uses directed moving nests and instead employs HWRF's original vortex-following strategy.

\section{b. GSI-based dual-resolution EnVar}

This section describes the general dual-resolution 4DEnVar form implemented in the GSI. Similar notations for dual-resolution EnVar can be found in Schwartz et al. (2015), Kleist and Ide (2015a,b), Schwartz (2016), and T. Lei and X. Wang (2017, unpublished manuscript). The description of the hybrid 4DEnVar formula mirrors those in Wang (2010) and Wang and Lei (2014). The analysis increment $\mathbf{x}_{t}^{\prime}$ at time $t$ within a DA window for the dual-resolution hybrid 4DEnVar is defined as

$$
\mathbf{x}_{t}^{\prime}=\mathbf{x}_{1}^{\prime}+\mathbf{D} \sum_{k=1}^{K}\left[\mathbf{a}_{k}^{\circ}\left(\mathbf{x}_{k}^{e}\right)_{t}\right]
$$

where $\mathbf{x}_{1}^{\prime}$ denotes the increment from the GSI static covariance, $\mathbf{D}$ denotes the operator that maps the fields from the coarser ensemble grids to the finer control grids, $\mathbf{a}_{k}$ denotes the augmented control vectors for the $k$ th ensemble member, $\left(\mathbf{x}_{k}^{e}\right)_{t}$ denotes the $k$ th ensemble perturbation 
at time $t$ normalized by $(K-1)^{1 / 2}, K$ is the ensemble size, and the symbol $\circ$ denotes the Schur product.

The cost function remains the same as in singleresolution 4DEnVar (Wang and Lei 2014):

$$
\begin{aligned}
J\left(\mathbf{x}_{1}^{\prime}, \mathbf{a}\right)= & \frac{1}{2} \beta_{1}\left(\mathbf{x}_{1}^{\prime}\right)^{\mathrm{T}} \mathbf{B}_{1}^{-1}\left(\mathbf{x}_{1}^{\prime}\right)+\frac{1}{2} \beta_{2}(\mathbf{a})^{\mathrm{T}} \mathbf{A}^{-1}(\mathbf{a}) \\
& +\frac{1}{2} \sum_{t=1}^{L}\left(\mathbf{y}_{t}^{o \prime}-\mathbf{H}_{t} \mathbf{x}_{t}^{\prime}\right)^{\mathrm{T}} \mathbf{R}_{t}^{-1}\left(\mathbf{y}_{t}^{o \prime}-\mathbf{H}_{t} \mathbf{x}_{t}^{\prime}\right),
\end{aligned}
$$

where $\mathbf{B}_{1}$ denotes the static covariance; $\mathbf{a}$ is a vector that concatenates $\mathbf{a}_{k}, k=1, K$; $\mathbf{A}$ denotes the matrix that defines the localization applied to the ensemble covariance; $L$ is the length of the DA window; and $\mathbf{R}_{t}, \mathbf{y}_{t}^{o \prime}$, and $\mathbf{H}_{t}$ refer to the observation error covariance, innovation vector, and linearized observation operator valid at time period $t$, respectively. Here $\beta_{1}$ and $\beta_{2}$ are the weights assigned for the static and ensemble covariance, respectively, where $\left(1 / \beta_{1}\right)+\left(1 / \beta_{2}\right)=1$ (Wang et al. 2008a,b).

The major difference between dual-resolution and single-resolution hybrid 4DEnVar is the incorporation of the operator $\mathbf{D}$, which allows the coarser-resolution ensemble to be projected to the finer control background. In other words, dual-resolution 4DEnVar formula returns to the single-resolution formula when the operator $\mathbf{D}$ is an identity matrix. Additionally, since the 4DEnVar is a temporal extension of 3DEnVar (Wang and Lei 2014), Eqs. (1) and (2) become dual-resolution 3DEnVar if a single time $t$ is considered in the DA window.

\section{c. EnKF}

As mentioned in section 2a, the GSI-based hybrid EnVar DA system for HWRF includes the HWRF EnKF component to provide ensemble background covariance during the continuous DA cycling. Following Lu et al. (2017), the EnKF component adopts the ensemble square root filter (EnSRF; Whitaker and Hamill 2002). As described in Lu et al. (2017), this EnKF code has been interfaced with HWRF and uses the observation preprocessing, quality control and forward operators provided by GSI. The cutoff radius for the covariance localization adopted by the EnKF is similar to that used by the EnVar component. Additionally, the relaxation to prior spread (RTPS) multiplicative inflation algorithm developed by Whitaker and Hamill (2012) is adopted. The details of the selected parameters are included in section $3 b$.

\section{d. Directed moving nest strategy}

As stated in section 1, a directed moving nest strategy is developed and applied to produce the first guess forecasts during the DA cycling. The primary objective of this development is to solve the issue associated with nonoverlapped ensemble domains for storm-following moving nests without needing DA code changes. In the traditional storm-following moving nest, the center of the nest for each ensemble member will follow the center of its own simulated TC. At the end of the first guess simulations, the ensemble nest locations diverge, which introduces complications for ensemble-based DA. In the new moving nest strategy, rather than letting the center of the domain follow its own simulated storm, the center of the domains for both the ensemble and the control background are moved to the same prescribed locations during model integration by adding a piece of code to the HWRF Model. This moving nest strategy has flexibility to determine the prescribed locations as well as the flexibility to prescribe a specific time period to move during the first guess forecast. For example, in order to implement FGAT or 4DEnVar from hours 3 to 9 , the domains can move for the first $3 \mathrm{~h}$ and stay in the same position during the remaining $6 \mathrm{~h}$ of model integration. This directed moving nest strategy is simple to implement without requiring changes in the DA code.

As described earlier, this newly developed directed moving nest strategy has the flexibility to choose the source of the prescribed locations. The prescribed locations determine the movement of the directed domains. Therefore, it is important to explore the sensitivity of this new directed moving nest strategy to the different sources of the prescribed locations. Additional experiments have been conducted with 3-h prescribed locations produced from interpolating and extrapolating TCVital, GFS forecasts, and blending the GFS forecast with extrapolated TCVitals. The locations produced by these methods are similar except for slight differences at the early stage of Edouard when the storm is relatively weak and disorganized (not shown). Given the limited differences in the prescribed locations from different sources, sensitivity experiments showed they also produce reasonably similar DA and forecast results with the different prescribed positions (not shown). This study uses interpolated TCVital for illustration, though extrapolated TCVital, 3-h global forecast, or the combination of the two can all be applied in operational settings.

Figure 2 illustrates the details of this directed moving nest strategy. At the initial time on 15 September 2014, all the domains overlap (Fig. 2a). After $3 \mathrm{~h}$ of integration (Fig. 2b), the nests of ensemble members using the storm-following moving nest strategy start to diverge, while the nests using directed moving nest strategy move toward the same location. Six hours later (Fig. 2c), the ensemble members using the storm-following strategy move farther apart, but those using the directed moving strategy remain in the same location. Although this strategy differs from that used by the operational HWRF, the forecast fields after $6 \mathrm{~h}$ of integration with each strategy are nearly identical, suggesting the 
(a) SLP@ 2014-09-15_06:00:00

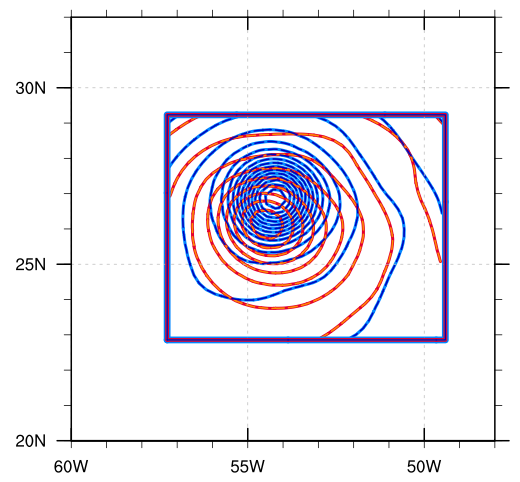

(b) SLP@ 2014-09-15_09:00:00

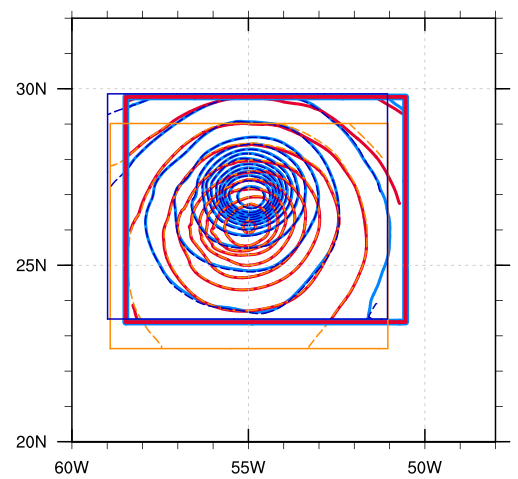

(c) SLP @ 2014-09-15_12:00:00

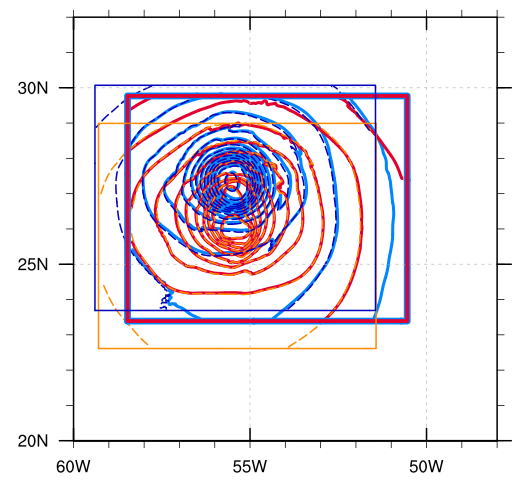

FIG. 2. Sea level pressure field at (a) +0000 , (b) +0300 , (c) +0600 UTC for the original HWRF moving strategy (dashed contour with thin box) and the directed moving strategy (solid contour with thick box) for ensemble member 1 (red and orange) and 2 (blue and cyan). Note that large areas of solid and dashed contours are overlain and indistinguishable due to the almost identical forecast fields.

directed moving nest method reconciles with the model integration as well as the storm-following moving nest.

\section{e. Integrating vortex relocation $(V R)$ and modification (VM) with hybrid DA}

The average location error in a 6-h background forecast is around 15-40 km (Trahan and Sparling 2012; Tong et al. 2014), which can be detrimental to assimilating of inner-core observations using ensemble-based DA that adopts the Gaussian error distribution assumption (e.g., Chen and Snyder 2007; Yang et al. 2013; Lu et al. 2017). Therefore, in the fully cycled hybrid DA system, for each DA cycle as described in section 2a, both the background ensemble and control forecasts are relocated before being updated by the EnKF or hybrid. Before VR, the locations toward which the background forecasts will move are determined first. The locations are determined by applying the EnSRF method (Whitaker and Hamill 2002) to a single-variable problem as follows:

1) Update the ensemble mean location:

$$
\overline{\mathbf{x}}^{a}=\overline{\mathbf{x}}^{b}+\mathbf{K}\left(\mathbf{y}-\mathbf{H} \overline{\mathbf{x}}^{b}\right),
$$

where $\overline{\mathbf{x}}^{a}$ denotes the updated ensemble mean location, $\overline{\mathbf{x}}^{b}$ is the mean of the first guess locations, and $\mathbf{K}$ is the traditional Kalman gain, which is computed as $\mathbf{K}=\mathbf{P}^{b} \mathbf{H}^{\mathrm{T}}\left(\mathbf{H} \mathbf{P}^{b} \mathbf{H}^{\mathrm{T}}+\mathbf{R}\right)^{-1}$. Note that Eq. (3) is applied on a scalar system so $\mathbf{P}^{b}$ is the location error variance estimated by the ensemble, $\mathbf{H}$ is a unit operator because the background ensemble contains only positions of hurricanes, $\mathbf{y}$ is the location observations obtained from TCVital, and $\mathbf{R}$ is the error variance of the TCVital locations. The standard error of the TCVital locations is estimated to be $10 \mathrm{~km}$ in this study. This estimation is based on and adapted from Trahan and Sparling (2012).
2) Update the location perturbations:

$$
\mathbf{x}_{k}^{\prime a}=(\mathbf{I}-\tilde{\mathbf{K}} \mathbf{H}) \mathbf{x}_{b}^{\prime k},
$$

where $\tilde{\mathbf{K}}=\left[1+\sqrt{\mathbf{R} /\left(\mathbf{H P}^{b} \mathbf{H}^{\mathrm{T}}+\mathbf{R}\right)}\right]^{-1} \mathbf{K}$ denotes the reduced Kalman gain, $\mathbf{I}$ is the identity matrix, and $\mathbf{x}_{k}^{\prime b}$ are the deviation of the $k$ th first guess location from the mean location and $\mathbf{x}_{k}^{\prime a}$ is the corresponding updated location perturbation.

3) Combine Eqs. (3) and (4) to get the updated ensemble of locations:

$$
\mathbf{x}_{k}^{a}=\overline{\mathbf{x}}^{a}+\mathbf{x}_{k}^{\prime a},
$$

where $\mathbf{x}_{k}^{a}$ is the $k$ th analyzed location.

After the locations are determined, the following relocation procedures are modified based on the HWRF VR procedure (Liu et al. 2000, 2006; Tallapragada et al. 2014) and are applied to relocate both the control and ensemble background. The HWRF forecast vortex is separated from its environment and placed in the location determined by either Eq. (3) or (5). The VM procedure is applied to the control vortices by adjusting the HWRF vortex size and intensity before putting it back into the HWRF environment. Because of the incongruences between VM and inner-core DA in updating the vortex size and intensity (e.g., Zhao et al. 2015), VM is only applied to the control background for the DA cycles when no inner core data (e.g., TDR data) are available.

\section{Experiment design}

\section{a. HWRF Model configuration}

The HWRF Model has been developed by the Environmental Modeling Center (EMC) in collaboration with the Geophysical Fluid Dynamics Laboratory (GFDL) and 
the University of Rhode Island (URI) since 2002 (Tallapragada et al. 2014). A nominal horizontal grid spacing of $0.18^{\circ} / 0.06^{\circ} / 0.02^{\circ}$ (approximately $27 / 9 / 3 \mathrm{~km}$ ) for the outermost/intermediate /innermost domains are used in this study, which is similar to that used in the 2014 operational HWRF. In this study, the intermediate and innermost domains adopt the strategies outlined in section 2 , while the outermost domain remains unmoved during the entire life of the storm. The outermost, intermediate, and innermost domains are configured with $216 \times 432$ horizontal grid points (approximately $80^{\circ} \times 80^{\circ}$ ), $232 \times 454$ horizontal grid points (approximately $30^{\circ} \times 30^{\circ}$ ), and $181 \times 322$ horizontal grid points (approximately $7^{\circ} \times 7^{\circ}$ ), respectively (Fig. 3). There are 61 vertical levels, and the model top is at $2 \mathrm{hPa}$ following $\mathrm{Lu}$ et al. (2017). The physics parameterization schemes used in HWRF Model follow those used in the 2014 operational HWRF except that the ocean coupling is turned off. Specifically, Ferrier microphysics (Ferrier 2005) as modified in HWRF for tropical applications, upgraded Simplified ArakawaSchubert (SAS) cumulus parameterization (Han and Pan 2011), HWRF surface layer parameterization, the GFDL slab scheme (Tuleya 1994), the HWRF PBL parameterization (Hong and Pan 1996), and Eta GFDL shortwave (Lacis and Hansen 1974) and longwave (Fels and Schwarzkopf 1975; Schwarzkopf and Fels 1991) radiation schemes are used to parameterize the microphysical, cumulus, surface layer, land surface, planetary boundary layer, and radiation processes. The cumulus scheme is not used in the innermost domain since the 3-km horizontal grid spacing is convection allowing. More details about these parameterization schemes can also be found in the HWRF scientific document by Tallapragada et al. (2014).

\section{b. Experiment setup}

To address the scientific questions proposed in section 1 , five experiments denoted as "3DEV," "3DEV-9kmS," "3DEV-NVRM," "3DEV-NEVR," and "4DEVTR" are conducted (see descriptions in Table 1). Details for each experiment are described as below.

3DEV is the baseline experiment, which performs 6-hourly, continuously cycled DA using 3DEnVar with FGAT following Fig. 1 and section $2 \mathrm{a}$. The newly developed directed moving nest strategy is adopted as introduced in section 2d. 3DEV starts at 1200 UTC 11 September 2014 when Edouard (2014) developed into a tropical depression. The first DA cycle starts at 1800 UTC 11 September 2014 after $6 \mathrm{~h}$ of spinup forecast initialized from the GFS analyses. The initial 6-h ensemble is initialized from the GFS ensemble as stated in section 2a. The DA experiment ends at 1800 UTC 19 September 2014 when Edouard became a low. A total of 32 DA cycles are performed in total.

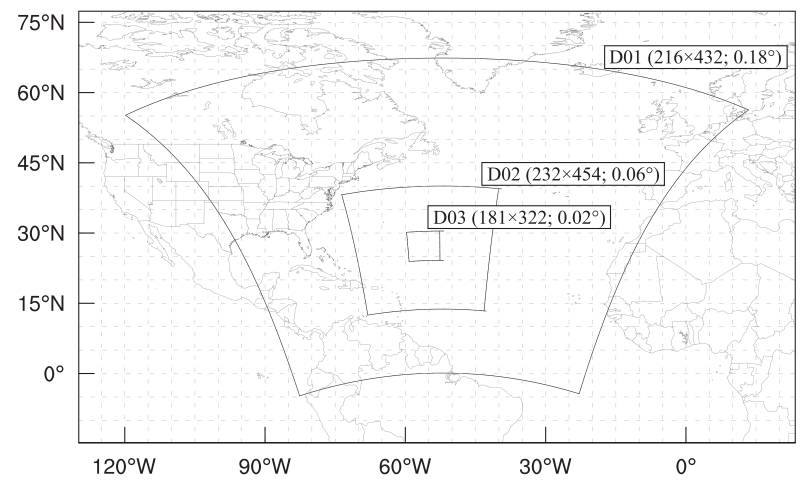

FIG. 3. Domain configuration example for Hurricane Edouard.

Assimilated observations include radial velocity from TDRs on board the NOAA P-3 aircraft (when available) in addition to conventional observations and clear-sky radiances from geostationary and polarorbiting satellites (Tallapragada et al. 2014). A description of these observations assimilated for each HWRF domain is given in Table 2. The general distribution and preprocessing for the TDR data can be found in Gamache (2005) and Lu et al. (2017). Although the satellite-derived wind and some in situ observations such as dropsonde, surface ship, and buoy observations are also assimilated, when it is available the TDR data play a dominant role in sampling the inner-core region. Specifically, the TDR data are available at the 16th, 17th, 21st, 24th, and 25th DA cycles valid at 1200 UTC 15 October, 1800 UTC 15 October, 1800 UTC 16 October, 1200 UTC 17 October, and 1800 UTC 17 October, respectively. The major difference between assimilation on the 3- and 9-km domains is that satellite radiances are not assimilated on the innermost domain. The bias corrections for the satellite radiances are estimated from the corresponding Global Data Assimilation System (GDAS) forecast.

Following Lu et al. (2017), an inflation factor of 0.9 is applied to the posterior ensemble variance to relax upon the prior ensemble variance during the DA cycling as proposed by Whitaker and Hamill (2012). For both the 3- and 9-km control analyses, the full ensemble error covariance is used in this study. This choice is motivated by the findings in Lu et al. (2017) that blending the static and flow-dependent ensemble covariance does not improve upon using the full ensemble covariance. This finding is consistent with Schwartz (2016), who found that blending the covariances may be detrimental when the resolution between static and ensemble error covariances is inconsistent. The horizontal and vertical localizations adopted in this study follow the configurations in 
TABLE 1. List of experiments and their descriptions.

\begin{tabular}{lcccccc}
\hline \hline Expt name & General features & Ensemble VR & $\begin{array}{c}\text { Control VM for } \\
\text { non-TDR }\end{array}$ & Control VR & $\begin{array}{c}\text { Dual } \\
\text { resolution }\end{array}$ & $\begin{array}{c}\text { 4DEnVar for } \\
\text { TDR cycles }\end{array}$ \\
\hline 3DEV & 6-hourly continuous & Yes & Yes & Yes & Yes & No \\
3DEV-9kmS & end-to-end cycling, & Yes & Yes & Yes & No & No \\
3DEV-NEVR & 3DEnVar hybrid & No & Yes & Yes & Yes & No \\
3DEV-NVRM & with FGAT, directed & No & No & No & Yes & No \\
4DEVTR & moving nest strategy & Yes & Yes & Yes & Yes & Yes \\
\hline
\end{tabular}

Lu et al. (2017). For the innermost and intermediate domains, $510-\mathrm{km}$ and 1.30 scale-height $e$-folding distances are used for the horizontal and vertical localizations, respectively.

The 3DEV-9kmS experiment is identical to 3DEV except there is no $3-\mathrm{km}$ innermost domain during DA. In other words, both the control analysis and the ingested ensemble use the 9-km horizontal grid spacing. The purpose of this experiment is to reveal the impact of introducing the 3-km horizontal grid spacing nest through dual-resolution DA in 3DEV. To isolate such impact, like 3DEV, 3DEV-9kmS still runs the 120 -h free forecast with a $27 / 9 / 3-\mathrm{km}$ triply nested configuration that is achieved by interpolating the $9-\mathrm{km}$ domain analysis to the innermost 3-km domain.

Experiments 3DEV-NEVR and 3DEV-NVRM examine the impacts of vortex initialization in this new DA system. 3DEV-NEVR is similar to 3DEV except that VR for the background ensemble is not performed. This experiment is meant to explore the necessity of doing VR for the ensemble background in the cycling hybrid DA system. 3DEV-NVRM follows the same procedures as 3DEV-NEVR, and the primary difference is that 3DEV-NVRM further removes the $\mathrm{VR}$ and VM procedures for the control background. In other words, 3DEV-NVRM performs neither VR nor $\mathrm{VM}$ for the control and ensemble backgrounds. The goal of this experiment is to investigate the necessity of doing VR or VM for the control member in the cycling hybrid DA system. Intercomparison among 3DEV, 3DEV-NEVR, and 3DEV-NVRM will reveal the relative importance of performing ensemble VR and VR/VM on the control member.

4DEVTR is identical to 3DEV in the DA cycles where TDR data are not available. In the cycles when TDR are available, 4DEnVar is applied in 4DEVTR for both the intermediate and innermost domains. The hourly control background and ensemble are provided for the 4DEnVar DA in this experiment following Wang and Lei (2014). The 4DEnVar is not applied to all the cycles because of the computational constraint. The primary goal of this experiment is to explore the impact of 4DEnVar for the innercore DA.

\section{Results}

a. Impact of incorporating the higher-resolution control analysis through dual-resolution hybrid $D A$

As stated earlier in section 1 and section $3 b$, in comparison with the configuration where all ensemble members are run at a high resolution, the dual-resolution configuration can save computational cost. In such a dualresolution configuration, only the control member is run at high resolution, and the rest of the ensemble members are still run at coarser resolution. Schwartz (2016) suggested the improvement of introducing a convectionallowing control member through dual-resolution DA for precipitation forecast over CONUS. Would introducing a single control member at a convection-allowing resolution improve the analysis and subsequent forecasts for hurricanes as well? Experiments 3DEV and 3DEV$9 \mathrm{kmS}$ are compared to address this question.

The vortex-scale wind structures in both the horizontal and vertical cross sections produced by the 3DEVand 3DEV-9kmS analyses are verified against the radar wind composite of Edouard by HRD. Figure 4 shows the model-derived wind and the corresponding verification at the 1-km height valid at 1800 UTC 15 September 2014. The verification is derived from the quality-controlled TDR radial velocity data by HRD (Gamache 2005; HRD radar wind data can be found at http://www.aoml.noaa. gov/hrd/Storm_pages/edouard2014/radar.html). The major difference in the analyses among $3 \mathrm{DEV}$ and $3 \mathrm{DEV}$ $9 \mathrm{kmS}$ is the depiction of the inner-core structures. In general, 3DEV fits the HRD composite better than $3 \mathrm{DEV}-9 \mathrm{kmS}$. Specifically, 3DEV can capture the dominant wind maximum in the northeastern quadrant present in the HRD composite (Figs. 4a,b). In comparison, the dominant wind maximum in $3 \mathrm{DEV}-9 \mathrm{kmS}$ is located in the southeastern quadrant. While $3 \mathrm{DEV}$ shows a circular structure consistent with the verifying wind analysis, the vortex in $3 \mathrm{DEV}-9 \mathrm{kmS}$ is more elliptical elongating 
TABLE 2. List of observations assimilated in each domain.

\begin{tabular}{|c|c|c|c|c|c|}
\hline & & & \multicolumn{3}{|c|}{ Domain } \\
\hline \multicolumn{3}{|c|}{ Data type } & $\begin{array}{c}\text { Outermost } \\
\text { domain }(\mathrm{d} 01)\end{array}$ & $\begin{array}{l}\text { Intermediate } \\
\text { domain }(\mathrm{d} 02)\end{array}$ & $\begin{array}{c}\text { Innermost } \\
\text { domain (d03) }\end{array}$ \\
\hline \multirow{10}{*}{$\begin{array}{l}\text { Conventional } \\
\text { observations }\end{array}$} & \multicolumn{2}{|c|}{ Radiosondes } & No observations & Yes & Yes \\
\hline & \multicolumn{2}{|c|}{ Dropwindsondes } & are assimilated and & Yes & Yes \\
\hline & \multicolumn{2}{|c|}{ Aircraft reports } & initialized from GFS & Yes & Yes \\
\hline & \multicolumn{2}{|c|}{$\begin{array}{l}\text { Surface ship and buoy } \\
\text { observations }\end{array}$} & & Yes & Yes \\
\hline & \multicolumn{2}{|c|}{$\begin{array}{l}\text { Surface observations } \\
\text { over land }\end{array}$} & & Yes & Yes \\
\hline & \multicolumn{2}{|c|}{ Pibal winds } & & Yes & Yes \\
\hline & \multicolumn{2}{|c|}{ Wind profilers } & & Yes & Yes \\
\hline & \multicolumn{2}{|c|}{$\begin{array}{l}\text { Radar-derived } \\
\text { velocity-azimuth } \\
\text { display wind }\end{array}$} & & Yes & Yes \\
\hline & \multicolumn{2}{|c|}{$\begin{array}{l}\text { WindSat scatter- } \\
\text { ometer winds }\end{array}$} & & Yes & Yes \\
\hline & \multicolumn{2}{|c|}{$\begin{array}{l}\text { Integrated pre- } \\
\text { cipitable } \\
\text { water derived } \\
\text { from the global } \\
\text { positioning system }\end{array}$} & & Yes & Yes \\
\hline \multicolumn{3}{|c|}{ Tail Doppler radar observation } & & Yes & Yes \\
\hline \multicolumn{3}{|c|}{ Satellite-derived wind } & & Yes & Yes \\
\hline \multirow{7}{*}{$\begin{array}{l}\text { Satellite } \\
\text { radiances }\end{array}$} & \multirow[t]{4}{*}{ IR } & Yes & & Yes & No radiances \\
\hline & & Yes & & Yes & are assimilated \\
\hline & & Yes & & Yes & \\
\hline & & Yes & & Yes & \\
\hline & \multirow[t]{3}{*}{ MW } & Yes & & Yes & \\
\hline & & Yes & & Yes & \\
\hline & & Yes & & Yes & \\
\hline
\end{tabular}

along the northwest-southeast axis. The size of the storm is also larger in $3 \mathrm{DEV}-9 \mathrm{kmS}$ in comparison with the verification and $3 \mathrm{DEV}$. In addition, the TC center as identified by the wind and pressure fields in $3 \mathrm{DEV}$ is more consistent with the best track center as compared with $3 \mathrm{DEV}-9 \mathrm{kmS}$.

Figure 5 shows a corresponding south-north vertical cross section of horizontal wind speed verification. The inner-core structure in $3 \mathrm{DEV}$ is again more consistent with the HRD radar wind composite than $3 \mathrm{DEV}-9 \mathrm{kmS}$. Specifically, though both $3 \mathrm{DEV}$ and $3 \mathrm{DEV}-9 \mathrm{kmS}$ capture the wind maximum peaks between $\sim 1$ and $2 \mathrm{~km}$ in the northern side of the cross section, the overall wind maximum is stronger in $3 \mathrm{DEV}$ than $3 \mathrm{DEV}-9 \mathrm{kmS}$. For example, the $50 \mathrm{~m} \mathrm{~s}^{-1}$ contour extends to $3.8 \mathrm{~km}$ in $3 \mathrm{DEV}$, which is closer to the verification, whereas the contour only extends to $2 \mathrm{~km}$ in $3 \mathrm{DEV}-9 \mathrm{kmS}$. In the southern side of the cross section, the wind maximum in $3 \mathrm{DEV}-9 \mathrm{kmS}$ is smoother and shallower compared to the HRD radar wind composite. In comparison, $3 \mathrm{DEV}$ shows greater spatial detail such as the narrowed peak of the $40 \mathrm{~m} \mathrm{~s}^{-1}$ contour above $3 \mathrm{~km}$ to the south, consistent with the HRD wind composite. Figure 5 also reveals a stronger and narrower warm core in $3 \mathrm{DEV}$ than $3 \mathrm{DEV}-9 \mathrm{kmS}$.

Additionally, the spatial correlation coefficient between the analyzed three-dimensional (3D) wind speed field and the HRD radar wind composite has been calculated and shown in Fig. 5 to provide a quantitative comparison following Lu et al. (2017). The higher correlation coefficient value in $3 \mathrm{DEV}(\sim 0.85)$ suggests a better analyzed storm inner-core structure as compared with $3 \mathrm{DEV}-9 \mathrm{kmS}$ (0.77). The average correlation coefficient for all the TDR cycles in $3 \mathrm{DEV}$ is 0.77 , and $3 \mathrm{DEV}-9 \mathrm{kmS}$ it is 0.72 (not shown). In summary, Figs. 4 and 5 show that the analyzed storm structure in 3DEV better agrees with the HRD composite than $3 \mathrm{DEV}-9 \mathrm{kmS}$, suggesting the advantage of incorporating a finer-resolution control analysis in the DA step through the dual-resolution EnVar capability.

In addition to the verification of the analyzed storm structures, the track, RMW, MSLP, and Vmax forecasts from all of Edouard's 32 cycles from 1200 UTC 11 September to 1200 UTC 19 September 2014 for Edouard (2014) are verified against the best track data (Fig. 6). Considering the limited samples collected from 
a) HRD radar@1 km $1800 Z 15$

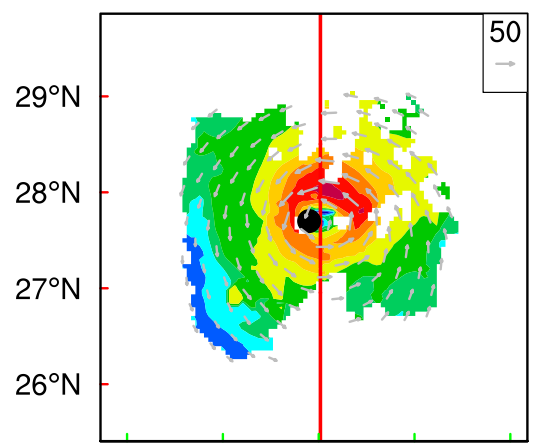

$58^{\circ} \mathrm{W} 57^{\circ} \mathrm{W} 56^{\circ} \mathrm{W} 55^{\circ} \mathrm{W} 54^{\circ} \mathrm{W}$ b) 3DEV @1 1km 1800Z15

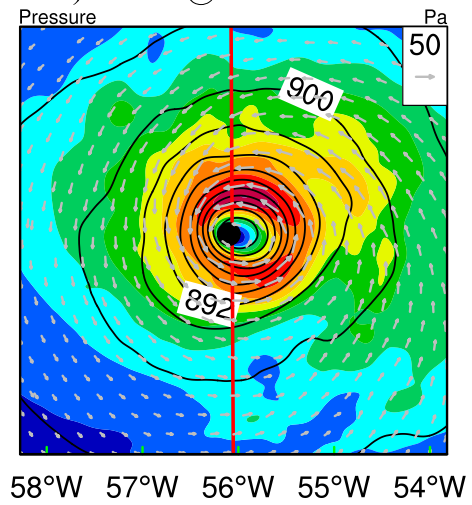

c) 3DEV-9kmS @, $1 \mathrm{~km} 1800 \mathrm{Z} 15$

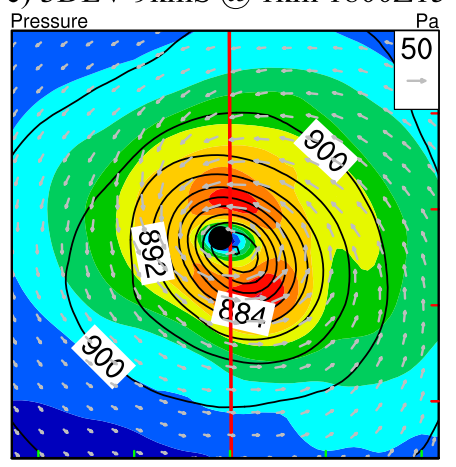

$58^{\circ} \mathrm{W} 57^{\circ} \mathrm{W} 56^{\circ} \mathrm{W} 55^{\circ} \mathrm{W} 54^{\circ} \mathrm{W}$

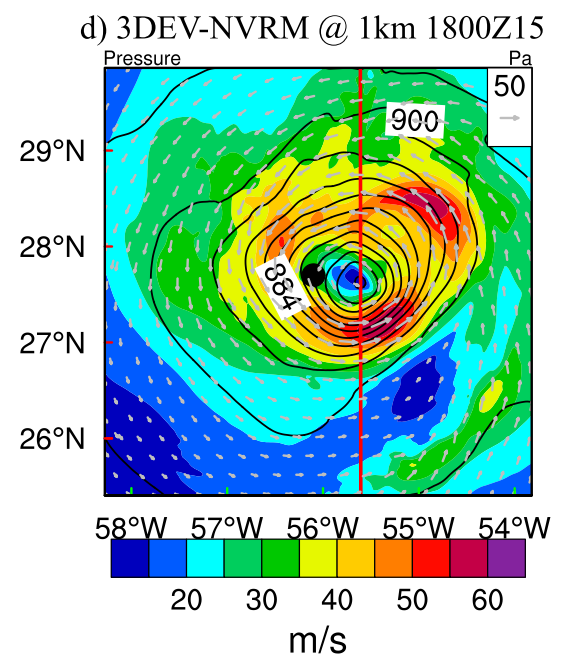

e) 3DEV-NEVR @1 $1 \mathrm{~km} \mathrm{1800Z15}$
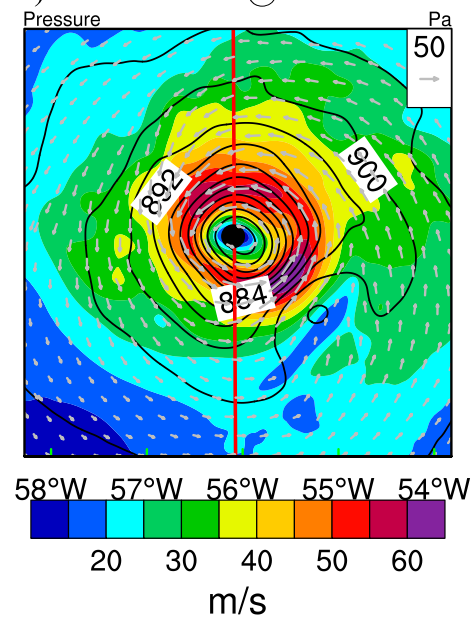

FIG. 4. Wind (shading and vectors) and pressure (contours) at 1-km height for (a) HRD radar wind composite, (b) 3DEV analysis, (c) 3DEV-9kmS analysis, (d) 3DEV-NVRM analysis, and (e) 3DEV-NEVR analysis for the second TDR mission valid at 1800 UTC 15 Sep 2014. The black dot is the best track position from NHC. The red line denotes the corresponding cross sections to be presented in Fig. 5.

the 32 cycles, the statistical significance of the differences between the experiments is assessed using a bootstrap resampling method following Davis et al. (2010). In this bootstrap resampling method, the resampling is performed on the differences of the absolute errors between a given pair of experiments from all cycles with replacement. A total of 10000 resamples were formed. The statistical significance level for each pair is defined as the rank where the resampled distribution crosses zero. The significance levels of $80 \%, 50 \%$, and $20 \%$ are marked with red lines in Figs. 6a-d.

According to Fig. 6, the profound difference between 3DEV and 3DEV-9kmS lies in the MSLP and Vmax forecasts at early lead times, and the experiments are overall comparable for both the track and RMW forecasts. For the mean absolute errors (Figs. 6c,d), 3DEV improves the MSLP and Vmax for the first $12-18 \mathrm{~h}$ compared to $3 \mathrm{DEV}-9 \mathrm{kmS}$. The smaller MSLP and
Vmax forecast errors at early lead times in 3DEV are direct results of more realistically analyzed inner-core structures than 3DEV-9kmS as revealed by Figs. 4 and 5 . In terms of error biases (mean error; Figs. 6e,f), 3DEV$9 \mathrm{kmS}$ produces large weak biases for both MSLP and Vmax at the initial time in comparison to the slight strong bias by $3 \mathrm{DEV}$. The weak MSLP bias in 3DEV is smaller through $87 \mathrm{~h}$, whereas the weak Vmax bias is comparable or slightly weaker in 3DEV-9kmS after $12 \mathrm{~h}$. This result suggests that there is possibly a systematic difference in the wind and pressure relationship between 3DEV-9kmS and 3DEV.

\section{b. Impact of vortex initialization (VI) and ensemble relocation}

As stated in the introduction, while recent studies show that efficient DA of dense inner-core observations can potentially replace the "bogus" VI to some extent 
a) HRD radar along lon $1800 \mathrm{Z} 15$

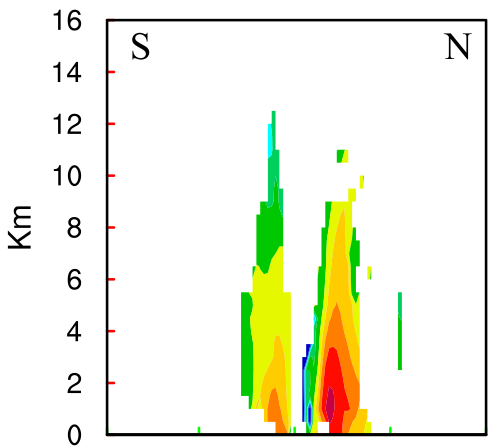

$225 \mathrm{Km} 12.5 \mathrm{Km}$ 0Km $112.5 \mathrm{KmR2} 2 \mathrm{Km}$ b) 3DEV@1800Z15

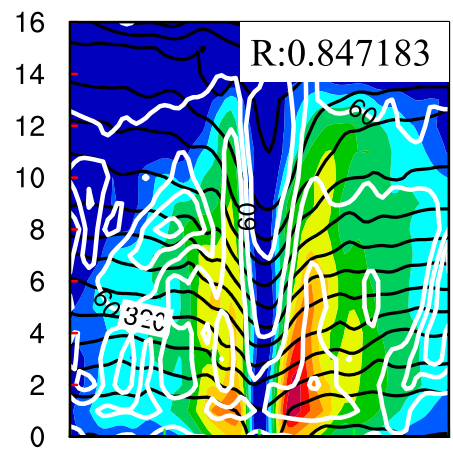

$224 \mathrm{Km} 112 \mathrm{Km} \quad 0 \mathrm{Km} \quad 112 \mathrm{Km} 224 \mathrm{Km}$ c) $3 \mathrm{DEV}-9 \mathrm{kmS} @ 1800 \mathrm{Z} 15$

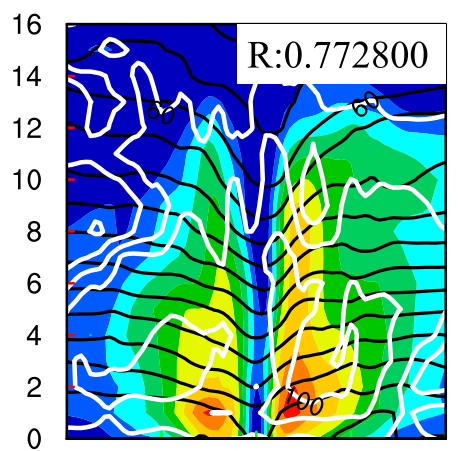

$224 \mathrm{Km} 112 \mathrm{Km} \quad 0 \mathrm{Km} \quad 112 \mathrm{Km} 224 \mathrm{Km}$

d) 3DEV-NVRM @ 1800Z15

e)3DEV-NEVR@1800Z15
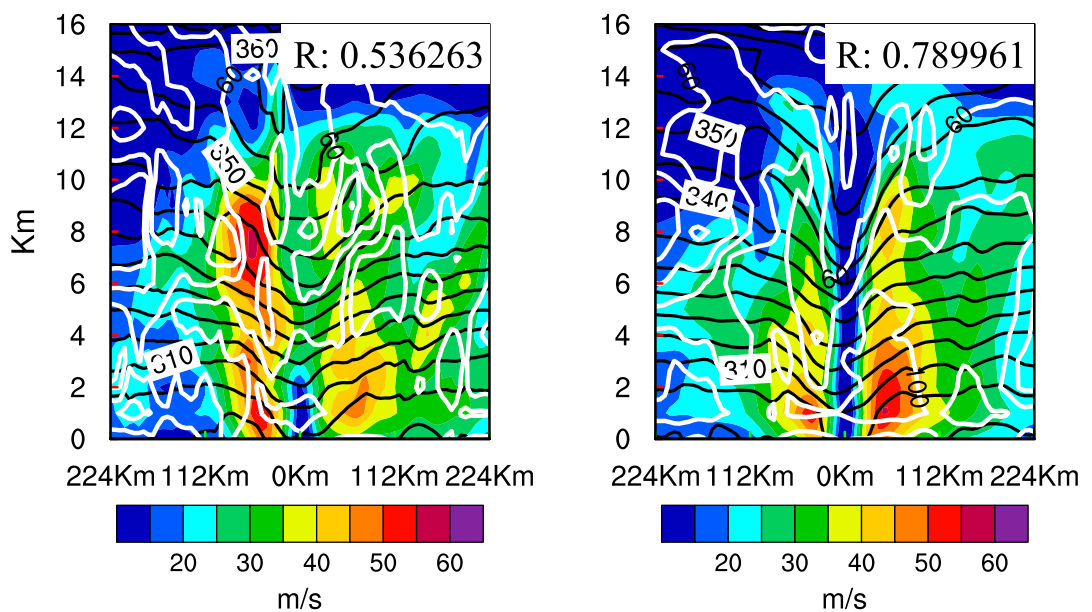

FIG. 5. Wind speed (shading), potential temperature (black contours), and relative humidity (white contours) analysis of the southnorth vertical section for (a) HRD radar wind analysis, (b) 3DEV, (c) 3DEV-9kmS, (d) 3DEV-NVRM, and (e) 3DEV-NEVR for the second TDR mission valid at 1800 UTC 15 Sep 2014. The values in (b)-(e) are the spatial correlation coefficients between the analyzed three-dimensional wind speed field and the HRD radar wind composite.

for hurricane prediction, these data are only available for a small part of the life cycle in most storms. Therefore, the VR and VM are likely necessary for a continuously cycled DA system when conventional inner-core observations are not available. Experiments are conducted in this subsection to reveal the impact of applying the VR and VM within an end-to-end, fully cycled hybrid DA system.

The analyses here show that VR and VM are most important in the control analysis, but applying VR to the background ensemble can also improve analyzed storm structure. In the horizontal structure analyses (Fig. 4), without applying any VR or VM, the wind structure analyses in 3DEV-NVRM largely disagree with the HRD composite (Fig. 4d). Specifically, the HRD radar composite contains one dominant wind maximum that is located in the northeast quadrant. In comparison, 3DEV-NVRM shows two comparable wind maxima located in the northeast and southeast quadrants, respectively. The northeast wind maximum in 3DEVNVRM is much farther away from the storm center compared to its counterpart in the HRD composite. In addition, a large location error is observed in 3DEVNVRM. Specifically, the 3DEV-NVRM shifts the storm center about $39 \mathrm{~km}$ to the east of the best track position. Compared to 3DEV-NVRM, 3DEV-NEVR reduces the location error and improves storm symmetry, which shows the benefit of applying the VR and VM to only the control background. Further applying VR to the background ensemble background improves the analysis even more. For example, two errors in 3DEV-NEVR (Fig. 4e) that are not present in $3 \mathrm{DEV}$ are a dominant wind maximum southeast of the storm and a dipole feature in the pressure field. These results are consistent with previous studies such as Chen and Snyder (2007) and Lu et al. (2017), which found dipolelike increments can occur when the storm locations prescribed in the background ensembles are too diverse or 
a) Mean Absolute Track Error

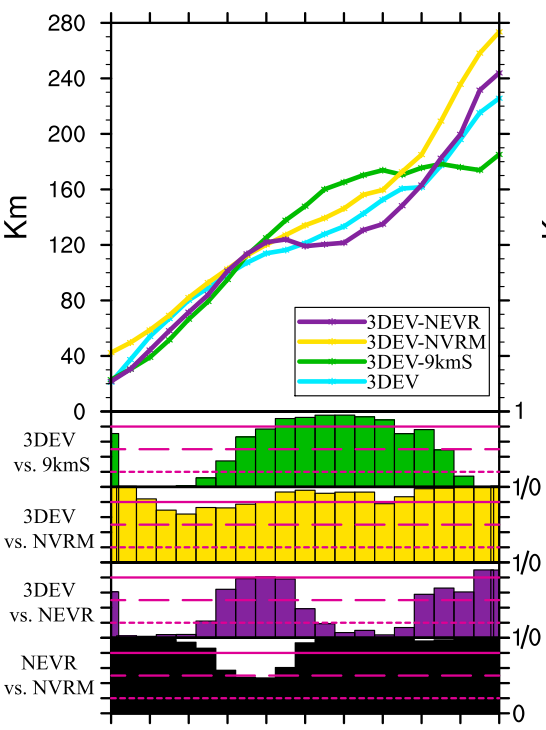

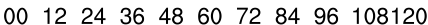
(32)(32)(32)(32)(32)(32)(31)(29)(27)(25)(23) c) Mean Absolute MSLP Error

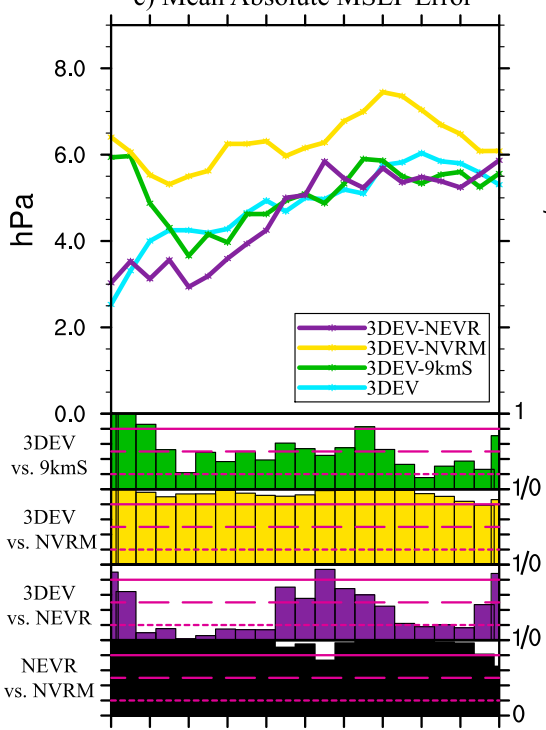

$\begin{array}{llllllllll}00 & 12 & 24 & 36 & 48 & 60 & 72 & 84 & 96 & 108120\end{array}$ (32)(32)(32)(32)(32)(32)(31)(29)(27)(25)(23) e) MSLP Error Bias

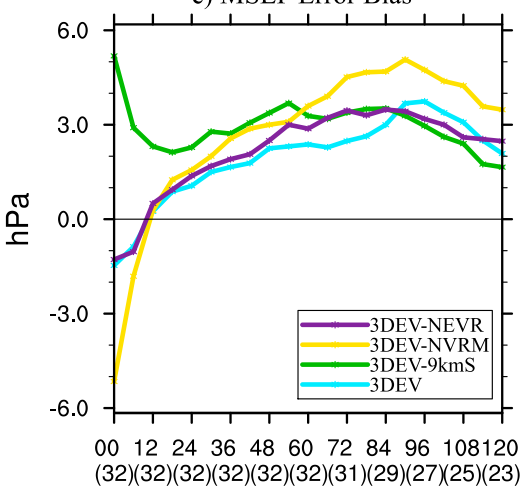

Forecast Hour b) Mean Absolute RMW Error

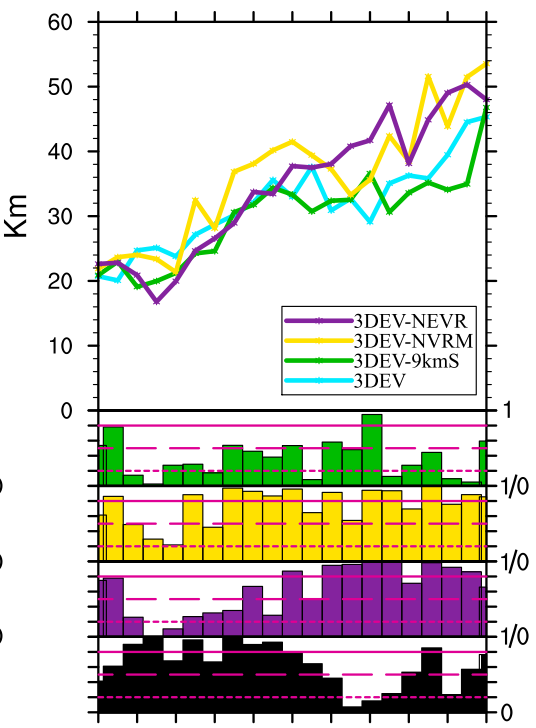

$00 \quad 12 \quad 24 \quad 36 \quad 48 \quad 60 \quad 72 \quad 8496 \quad 108120$

(32)(32)(32)(32)(32)(32)(31)(29)(27)(25)(23)

d) Mean Absolute Vmax Error

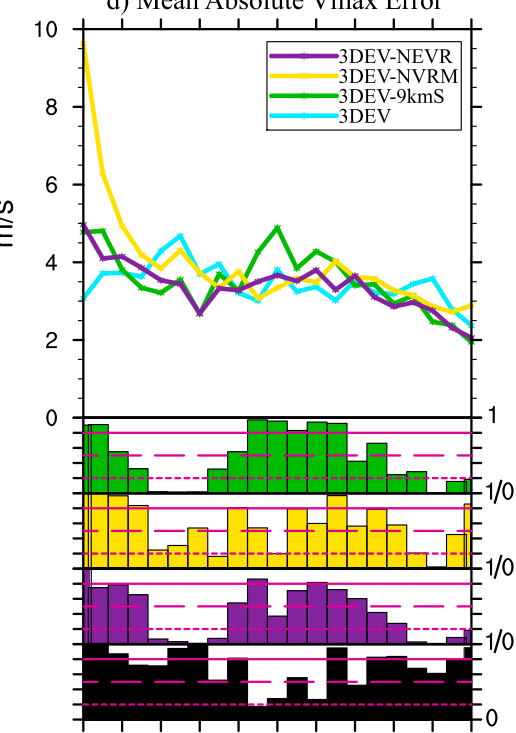

$0012 \quad 24 \quad 36 \quad 48 \quad 6072 \quad 8496 \quad 108120$ (32)(32)(32)(32)(32)(32)(31)(29)(27)(25)(23) f) Vmax Error Bias

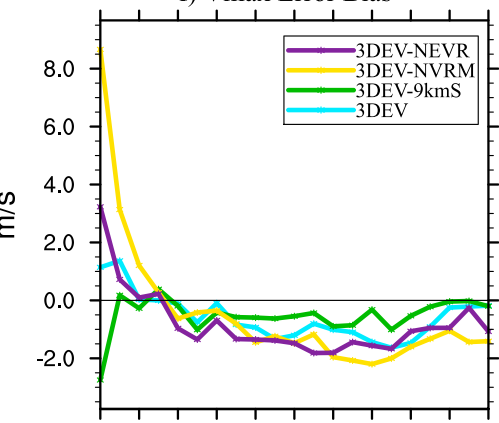

$0012 \quad 2436 \quad 48 \quad 6072 \quad 8496 \quad 108120$ (32)(32)(32)(32)(32)(32)(31)(29)(27)(25)(23)

Forecast Hour 
the deviation of the background storm location from the observed storm location is too large.

Vertical cross sections similarly show that VR and VM are most important for the control analysis. The innercore structure analyzed by 3DEV-NVRM (Fig. 5d) is also largely inconsistent with the HRD composite (Fig. 5a) with a much weaker wind maximum on the north side and a much stronger, deeper wind maximum on the south side. In comparison with 3DEV-NVRM, both the northern and southern wind maxima in 3DEV-NEVR are more consistent with the HRD composite in terms of both the vertical extent and magnitude. However, when compared to 3DEV, 3DEV-NEVR degrades the analysis. For example, the low-level wind maxima in $3 \mathrm{DEV}$ are more consistent with the verifying analysis.

The spatial correlation between the various analyses here and the HRD wind speed analysis objectively verifies the above conclusions. 3DEV, 3DEV-NEVR, and 3DEV-NVRM are correlated with the HRD analysis at $\sim 0.85, \sim 0.79$, and $\sim 0.54$, respectively. Furthermore, over all TDR cycles (not shown) the average correlation is $0.77,0.71$, and 0.53 , respectively. In summary, the results from both the horizontal and vertical structure analyses show that conducting VR and VM on the control background plays important roles in defining the location and structure of the analyzed storm. Further conducting VR in the ensemble background is also helpful in improving the structure of the analyzed storm. However, it should also be noted that the importance of ensemble VR may not be as important as the control VR and VM.

The mean absolute forecast errors in 3DEV-NVRM are much larger at most times than those in $3 \mathrm{DEV}$ (Figs. 6a-d). Without any VR or VM, the track, RMW, MSLP, and Vmax forecasts produced by 3DEV-NVRM perform the worst. Consistent with Figs. $4 d$ and 5d, the significantly larger track error at the early lead times in 3DEV-NVRM results from large location errors in the analysis. The overall larger RMW mean absolute errors in 3DEV-NVRM than 3DEV can possibly be attributed to the unrealistically analyzed storm structure as shown in Figs. $4 \mathrm{~d}$ and $5 \mathrm{~d}$ where multiple wind maxima exist at different heights and distances. The highly asymmetric storm in 3DEV-NVRM analysis is also consistent with significantly larger MSLP error for all lead times and significantly larger Vmax errors in the first $21 \mathrm{~h}$ in the 3DEV-NVRM forecast (Figs. 6c,d). Forecast error biases (Figs. 6e,f) show that 3DEV-NVRM has a much larger strong bias for both MSLP and Vmax than 3DEV at the initial time. At later lead times after about 12-36h, 3DEV-NVRM shows larger weak bias compared to 3DEV. The large bias transition at about 12-21-h lead times implies a significant wind and pressure field adjustment by 3DEV-NVRM.

The forecasts in 3DEV-NEVR are better than those produced by 3DEV-NVRM. Specifically, 3DEV-NEVR produces a better track and MSLP forecast at all lead times and a better RMW and Vmax forecasts at most lead times. This result differs from the early study by Schwartz et al. (2013), who found VR applied for the control member did not improve cycling hybrid DA. Such different results could be possibly due to the continuous location correction by ingesting bogus innercore observations during all cycles in Schwartz et al. (2013), while this study uses no bogus data with innercore real observations available only at limited times.

Meanwhile, the performance of the track, RMW, MSLP, and Vmax forecasts by 3DEV-NEVR and 3DEV are commensurate over the entire 5-day forecast period (Figs. 6a-d), though 3DEV-NEVR appears to be right for the wrong reason at times. Further examination reveals that 3 DEV-NEVR begins with a strong bias

FIG. 6. (a) Mean absolute track forecast error, (b) mean absolute RMW forecast error, (c) mean absolute MSLP forecast error, and (d) mean absolute 10-m Vmax forecast error for 32 missions of Edouard (2014) for 3DEV (turquoise), 3DEV-9kmS (green), 3DEVNVRM (yellow), and 3DEV-NEVR (purple). (e),(f) The corresponding MSLP and Vmax forecast error bias, or mean error. The green, yellow, and purple histograms below (a) (d) are the bootstrap significance levels where $3 \mathrm{DEV}$ is statistically different from 3DEV-9kmS (A: "3DEV" minus B: "3DEV-9kmS"), 3DEV-NVRM (A: "3DEV", minus B: "3DEV-NVRM"), and 3DEV-NEVR (A: "3DEV” minus B: “3DEV-NEVR"), respectively. The black histograms denote the bootstrap significance levels where 3DEVNEVR is statistically different from 3DEV-NVRM (A: "3DEV-NEVR" minus B: "3DEV-NVRM"). The significance levels of $80 \%$ (solid) and $20 \%$ (dotted) for each pair are given as red lines in the histograms to indicate where $\mathrm{A}$ is significantly better or worse than B, respectively. The significance levels between $20 \%$ and $80 \%$ indicate that there is no statistically significant difference between A and B. The significance level of $50 \%$ is also shown with red dashed lines to indicate if $\mathrm{A}$ is better $(>50 \%)$ or worse $(<50 \%)$ than $\mathrm{B}$. The numbers in the brackets under the $x$ axis are the number of cases at each lead time. 
a) HRD radar @ 1 km $15 Z 17$

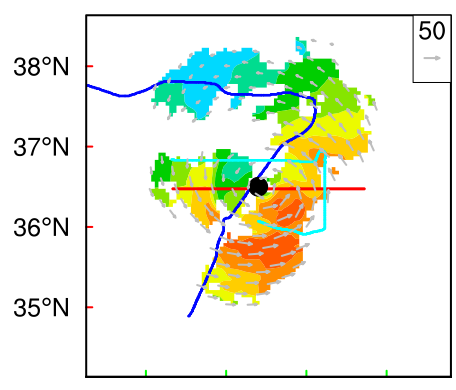

$55^{\circ} \mathrm{W} \quad 54^{\circ} \mathrm{W} \quad 53^{\circ} \mathrm{W} \quad 52^{\circ} \mathrm{W}$

d) HRD radar along lat @ 15Z17

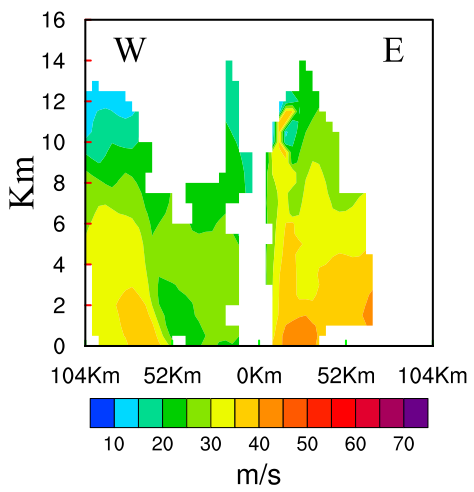

b) 3DEV @ 1km $12 \mathrm{Z17}$

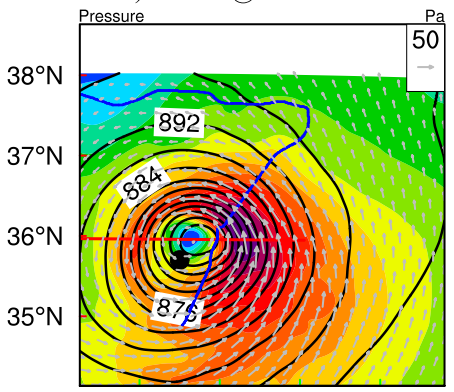

55 W 54 W 53 w 52 w

e) 3DEV @12Z17

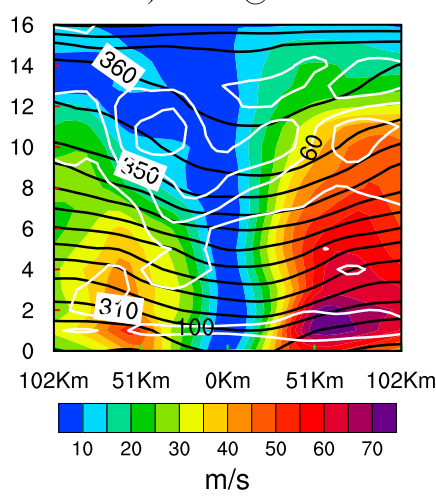

c) 4DEVTR@1km $12 \mathrm{Z} 17$

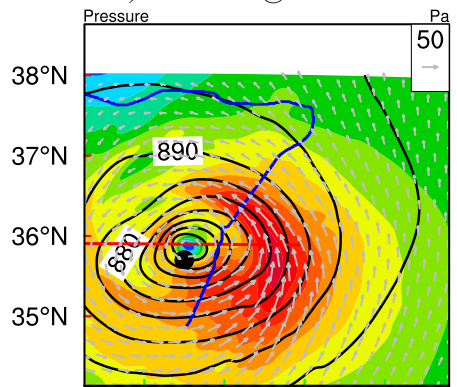

$55^{\circ} \mathrm{W} 54^{\circ} \mathrm{W} 53^{\circ} \mathrm{W} 52^{\circ} \mathrm{W}$

f) 4DEVTR @ $12 \mathrm{Z17}$

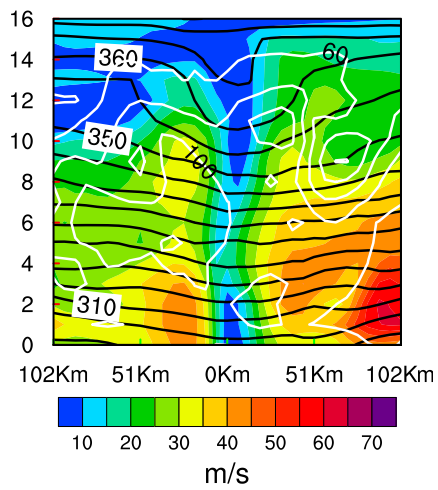

FIG. 7. Horizontal plots of (a)-(c) wind (shading and vectors) and pressure (contours) at 1-km height as well as west-east cross sections of (d)-(f) wind speed (shading), potential temperature (black contours), and relative humidity (white contours) for the (left) HRD radar wind composite, (middle) 3DEV, and (right) 4DEVTR for the fourth TDR-involved cycle valid at 1200 UTC 17 Sep 2014. The west-east vertical cross sections in (d)-(f) are denoted by the corresponding red lines in (a)-(c). The blue line in (a)-(c) denotes the flight track when TDR data were available between 1258 and 1417 UTC, and the turquoise line in (a) denotes the flight track when TDR data were available between 1617 and 1708 UTC. Because of the data distribution, the HRD radar composite is valid at 1500 UTC 17 Sep 2014.

followed by an incorrect intensification rate as implied by the evolution of bias (too slow, e.g., Fig. 6f). These errors effectively cancel, leading to a better fit of 3DEV-NEVR to the best track during the 18-48-h lead times (e.g., Figs. 6c,d).

\section{c. Impacts of 4DEnVar for vortex-scale observation assimilation}

As stated in section 1, hybrid 3DEnVar DA with a 6-h time window is unlikely to resolve quickly evolving storm features due to the inability of 3DEnVar to capture the temporal evolution of error covariance in a 6-h DA window. However, 4DEnVar might alleviate this issue because of its design (section 3b). To test this hypothesis, TDR DA experiments are first conducted for the cycles when Edouard was going through the rapid eyewall replacement.

The final NOAA P3 TDR mission in Edouard occurred during the eyewall replacement or the end of the "decaying double eyewall storm" period (Abarca et al. 2016). During this time period, Edouard was weakening, likely due to the eyewall replacement and cold upwelling or mixing (Stewart 2014). One NOAA P3 aircraft was sent out for HRD-tasked model evaluation between 1110 and 1919 UTC 17 September 2014, but its data were unfortunately divided between the 1200 and 1800 UTC cycles, yielding a brief and uneven distribution in each individual cycle. The briefly and unevenly distributed data further increase the potential benefit of 4DEnVar over 3DEnVar DA as stated in the introduction. The temporal coverage of the TDR data is between 1258 and 1417 UTC for the 1200 UTC cycle and between 1617 and 1708 UTC for the 1800 UTC cycle. The spatial coverage of the TDR data is added to Fig. 7a.

The hybrid 4DEnVar analysis in 4DEVTR better captures the structure of Edouard during this period. Figure 7 compares 1-km winds (Figs. 7a-c) and a vertical cross section of winds (Figs. 7d-f) with the HRD radar composite. The HRD radar composite shows a wind maximum southeast of the storm center as well as a secondary wind maximum east of the storm center. The vertical cross section in Fig. $7 \mathrm{~d}$ captures the edge of the 
inner wind maximum between $\sim 1$ and $35 \mathrm{~km}$ east of the storm center as well as the complete outer maximum at $60 \mathrm{~km}$. Compared with the HRD radar composite, 3DEV contains a spuriously strong wind maximum $\sim 50-75 \mathrm{~km}$ east of the storm (Figs. 7b,e), while 4DEVTR has two maxima roughly at the correct locations (Figs. 7c,f).

Likewise, the MSLP and Vmax forecasts initialized from the 4DEVTR analysis for this cycle are more consistent with the best track than the forecasts initialized from the 3DEV analyses, especially at early lead times (not shown). Consistent analysis and forecast performance in 4DEVTR and $3 \mathrm{DEV}$ are also found in the next consecutive cycle valid at 1800 UTC (not shown). This result suggests the benefit of utilizing 4DEnVar over 3DEnVar in the situation of rapidly evolving error covariance and temporally unevenly distributed observations.

Figures 8a-d show the track, RMW, MSLP, and Vmax forecast absolute errors over all cycles for the 4DEVTR and the 3DEV experiments. From section 3b, the only difference between 4DEVTR and 3DEV is the DA method applied for the TDR cycles. Therefore, by design, 4DEVTR reveals the direct impact of 4DEnVar on analyses and forecasts where TDR was available and also the secondary impact on subsequent analyses and forecasts. The performance of track forecasts by $3 \mathrm{DEV}$ and 4DEVTR are comparable although slight but statistically significant degradation is found in 4DEVTR at longer lead times (Fig. 8a). The RMW absolute forecast error is also in general comparable between $3 \mathrm{DEV}$ and 4DEVTR except for the first $18 \mathrm{~h}$. This initial RMW degradation in 4 DEVTR is primarily found during the "decaying double-eyewall" stage of Edouard (Abarca et al. 2016). Although the double eyewall is correctly captured by 4DEVTR and missed by 3DEV during this stage, sometimes 4DEVTR places a slightly larger wind maximum to the secondary eyewall rather than the primary eyewall, which leads to a larger RMW (not shown).

The main forecast improvement in 4DEVTR comes at early lead times. Relative to 3DEV, 4DEVTR measured in absolute errors, significantly improved MSLP forecasts for the first $15 \mathrm{~h}$ and also Vmax forecasts for the first $45 \mathrm{~h}$ (Figs. 8c,d). The MSLP and Vmax improvements at the early lead times for 4DEVTR are consistent with the better analyses that greatly reduced spurious wind maxima (e.g., Figs. 7 and 8f). The results are mixed at later lead times. Biases in 3DEV of MSLP and Vmax appear to benefit from compensating errors (i.e., strong initial bias followed by an unrealistically slow intensification rate). As shown in Figs. 8e and 8f, 4DEVTR has a much smaller bias at the initial time yet a similarly erroneous intensity evolution as $3 \mathrm{DEV}$. This results in 4DEVTR having larger bias values from 6- to 12-h lead time through about 66-78 h.

\section{d. Investigation of intensity forecast improvement over the operational HWRF}

As stated in the introduction, this subsection explores the potential of the newly developed DA system to improve the intensity forecast by comparing the analyzed TC structure with that from the operational HWRF where negative Vmax bias occurred during the intensification of Edouard.

Figure 8 shows the track, RMW, MSLP, and Vmax forecast errors averaged over the 32 cycles from the operational HWRF. The performance of track forecasts measured in absolute errors between 4DEVTR and the 2014 operational HWRF are in general mixed, where 4DEVTR performs better in the later lead times and worse in the earlier lead times. 4DEVTR generally produces better size forecasts as compared to the operational HWRF except the first $18 \mathrm{~h}$ (Fig. 8b). The slightly larger initial RMW of 4DEVTR has been discussed in section $4 \mathrm{c}$ due to the ambiguity of identifying the RMW when a realistic double eyewall is captured in 4DEVTR. Significant improvements in both MSLP and Vmax forecasts are found in 4DEVTR compared to the operational HWRF in terms of both absolute errors and biases (Figs. 8c-f). The only exception is the slightly worse Vmax at the analysis time, which can be attributed to not using VI for the TDR-involved cycles in 4DEVTR. Specifically, 4DEVTR improves the 0-120-h MSLP forecast and the 8-120-h Vmax forecasts as compared with the operational HWRF.

Figure 9, which superposes all the deterministic MSLP and Vmax forecasts during Edouard (Figs. 9a,b), shows that the improvements in the forecasts from 4DEVTR primarily occur during the intensifying period. ${ }^{3,4}$ Figures $9 \mathrm{c}$ and $9 \mathrm{~d}$ show that 4DEVTR significantly alleviated the weak biases in the operational HWRF that resulted from slow or delayed intensification early in the forecast. Examples of two consecutive DA cycles during the intensifying period from 0000 and 0600 UTC 14 September 2014 are given in Figs. 9e-h. Since 4DEVTR uses the same physics as the operational HWRF (except the ocean coupling, which testing revealed to not impact results, not shown), the improvement seen here is likely a result of the upgraded DA system relative to the operational HWRF.

\footnotetext{
${ }^{3}$ Following Rogers et al. (2013a), an "intensifying" period is one in which the 12-h best track Vmax change is greater than $20 \mathrm{kt}$ $(24 \mathrm{~h})^{-1}\left[\approx 10.3 \mathrm{~m} \mathrm{~s}^{-1}(24 \mathrm{~h})^{-1}\right]$. Given this definition, the intensifying period is identified from 1200 UTC 13 September to 0600 UTC 15 September 2014.

${ }^{4}$ The first TDR mission was after this period.
} 
a) Mean Absolute Track Error

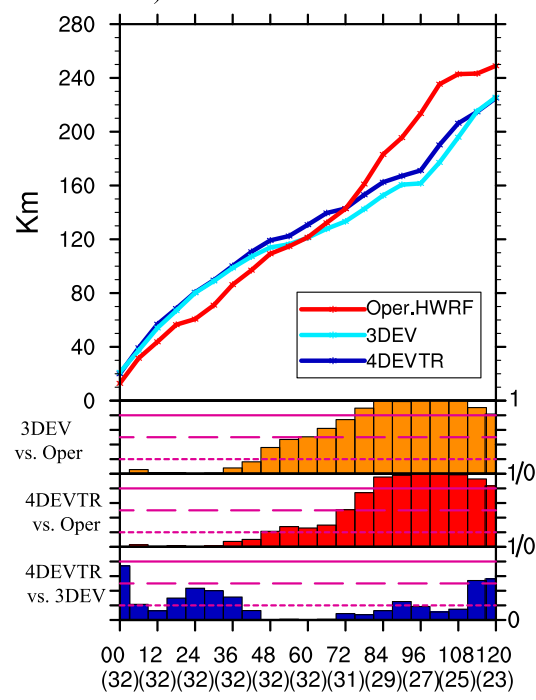

c)

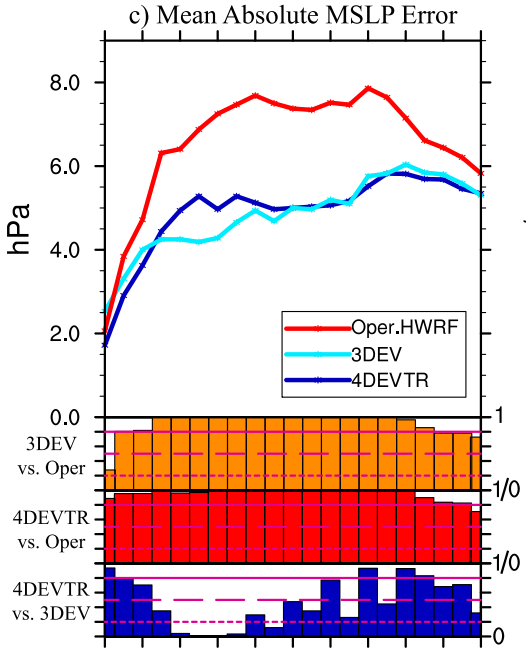

$0012 \quad 24 \quad 36 \quad 48 \quad 6072 \quad 8496 \quad 108120$

(32)(32)(32)(32)(32)(32)(31)(29)(27)(25)(23)

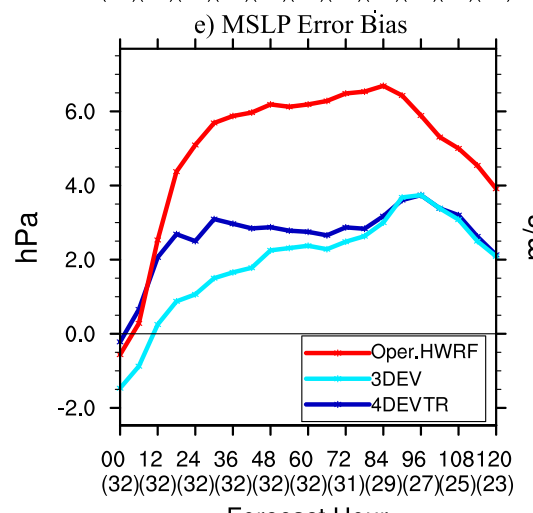

b) Mean Absolute RMW Error

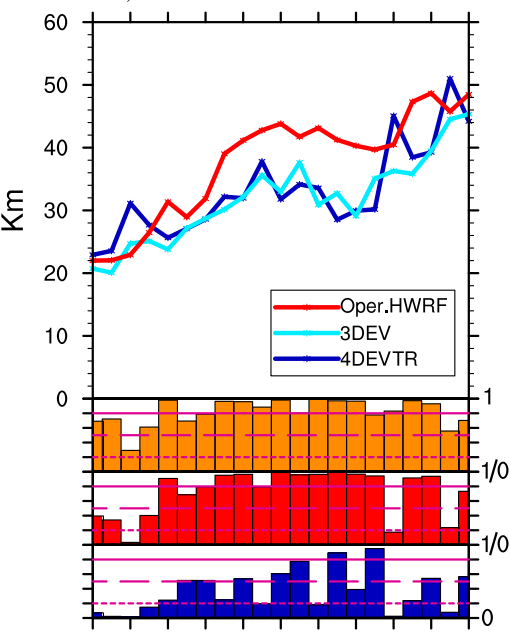

$0012 \quad 24364860728496108120$

(32)(32)(32)(32)(32)(32)(31)(29)(27)(25)(23)

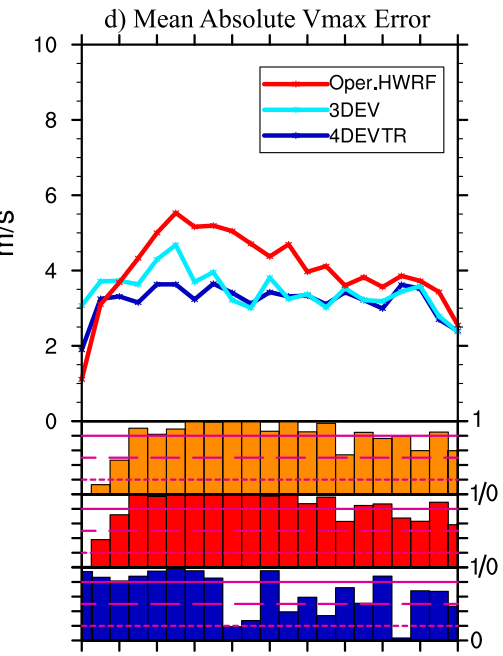

$0012 \quad 2436486072 \quad 8496108120$ (32)(32)(32)(32)(32)(32)(31)(29)(27)(25)(23)

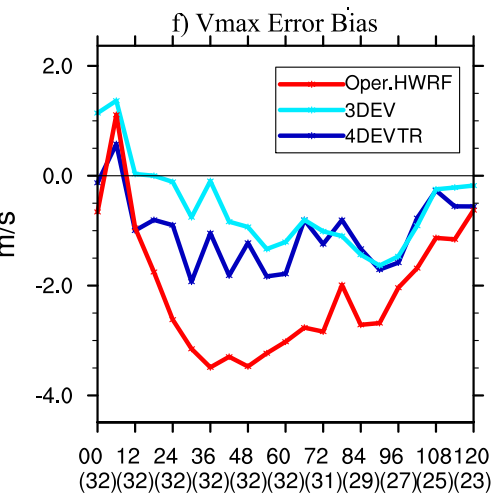

Forecast Hour

FIG. 8. As in Fig. 6, but for 3DEV (turquoise), 4DEVTR (blue), and the operational HWRF (red). The orange histograms below (a)-(d) are the bootstrap significance levels where 3 DEV is statistically different from the operational HWRF (A: "3DEV" minus B: "Oper.HWRF"). The red and blue histograms denote the bootstrap significance levels where 4DEVTR is statistically different from the operational HWRF (A: "4DEVTR" minus B: "Oper.HWRF") and 3DEV (A: “4DEVTR" minus B: “3DEV”). 
Figure 10 shows the time-averaged azimuth mean of inertial stability, radial wind, and tangential wind for the analyses and 12-h forecasts during the intensifying period of Edouard for both 4DEVTR and the operational HWRF. The inertial stability is calculated following Schubert and Hack (1982). In general, the analyses from 4DEVTR (Fig. 10a) have stronger inertial stability, a smaller RMW, shallower inflow depth, and stronger tangential wind compared with the analyses from the operational HWRF (Fig. 10d). The high inner-core inertial stability analyses are known to favor intensification (e.g., Schubert and Hack 1982; Holland and Merrill 1984; Hack and Schubert 1986), and the consistently strong inertial stability in 4DEVTR during the first $12 \mathrm{~h}$ is consistent with a continuous increase of the intensity of the storm initialized by 4DEVTR during the intensifying period of Edouard. These features are also consistent with findings from individual cycles such as those in Fig. 9. On the contrary, the analyses from the operational HWRF show weaker inertial stability, larger RMW, and weaker tangential wind in general (Fig. 10d). Therefore, the storm analyzed by the operational HWRF is less likely to produce immediate intensifications compared to 4DEVTR.

Additionally, similar Vmax but weaker inertial stability in the operational HWRF as compared to 4DEVTR indicates the nature of the vortices from the operational HWRF is inherently different. Fourier decomposition diagnostics were therefore performed on the absolute vorticity in the analyses following Reasor et al. (2000) to illustrate this point. The averaged percentage of variance explained by each wavenumber within the 1.5 RMW range over all model levels during the intensifying period is shown in Table 3. Larger percentages on relatively high wavenumbers indicate more asymmetric TC vortices. Table 3 thus indicates that the analyzed operational HWRF vortices are less symmetric than those from 4DEVTR. These asymmetric anomalies possibly contribute to the delayed intensification in the operational HWRF (e.g., Nolan and Montgomery 2002; Nolan and Grasso 2003; Nolan et al. 2007).

\section{Summary and conclusions}

A GSI-based, continuously cycled, dual-resolution hybrid EnKF-Var DA system is developed for HWRF with a self-consistent HWRF EnKF ensemble and a newly developed directed moving nest strategy. Both the hybrid 3DEnVar and hybrid 4DEnVar capabilities have been implemented with the system. The system also integrates with VM and VR that are used in the operational HWRF. In this paper, the newly developed hybrid DA system is first described. Using
Hurricane Edouard (2014), a few scientific questions discussed in section 1 are investigated by multiple sets of experiments.

In the baseline 3DEV experiment, 3DEnVar with FGAT is applied every $6 \mathrm{~h}$ continuously from the beginning to the end of Edouard (2014), assimilating the TDR radial velocity observations on board NOAA P-3 aircraft in addition to all in situ and satellite radiance observations from the operational HWRF data stream. Dual-resolution 3DEnVar is implemented with a control analysis at 3-km horizontal grid spacing and a selfconsistent HWRF EnKF ensemble at 9-km horizontal grid spacing. 3DEV adopts the newly developed directed moving nest strategy within continuous DA and forecast cycling associated with moving nests. VR is applied for both the control and ensemble background forecasts, and VM is applied to the control background in this experiment where TDR is not available. All other experiments are identical to $3 \mathrm{DEV}$ except each has major difference to address the scientific questions.

To investigate the impact of introducing a highresolution nest with dual-resolution DA in the baseline $3 \mathrm{DEV}$ experiment, $3 \mathrm{DEV}-9 \mathrm{kmS}$ is conducted where both the control and the ensemble background are run at 9-km horizontal grid spacing only. With the high-resolution control analysis, 3DEV produces storm structures with more detail and in better agreement with the observations as compared with $3 \mathrm{DEV}-9 \mathrm{kmS}$. The subsequent forecast suggests that the better analysis in 3DEV provides better subsequent MSLP and Vmax forecasts measured in both absolute errors and biases at early lead times compared to 3DEV-9kmS. These improvements seem to be short lived. The track and RMW forecasts are not improved by introducing the highresolution control analysis.

Two experiments were designed and conducted to investigate the importance of integrating VR and VM in the cycled hybrid DA system. Completely removing VR and VM (3DEV-NVRM vs 3DEV-NEVR) significantly degrades the TC analysis, the subsequent track, Vmax, and MSLP forecasts. Compared to not applying VM and VR at all (3DEV-NVRM), applying VR to the control background for all DA cycles and applying VM to the control background to the DA cycles without TDR (3DEV-NEVR) significantly improves the TC structure analysis, overall track forecasts, and RMW forecasts for the first $72 \mathrm{~h}$, MSLP forecast out to $120 \mathrm{~h}$, and Vmax forecast for the first $51 \mathrm{~h}$. Applying additional VR for the ensemble background forecasts improves the TC structure analysis further (3DEV). Specifically, the wind maxima are located in the quadrant that is consistent with the verifying observations, and spurious double SLP minima no longer exist. The subsequent short-term 
a) MSLP forecasts for all

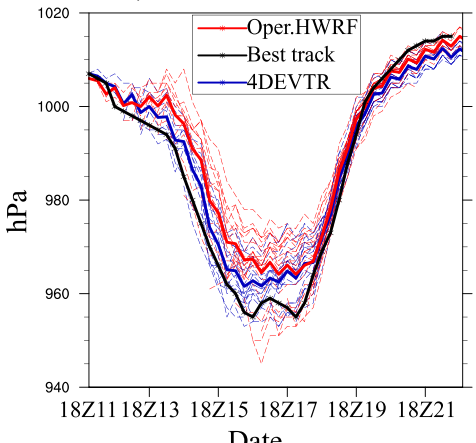

c) MSLP bias during intensifying

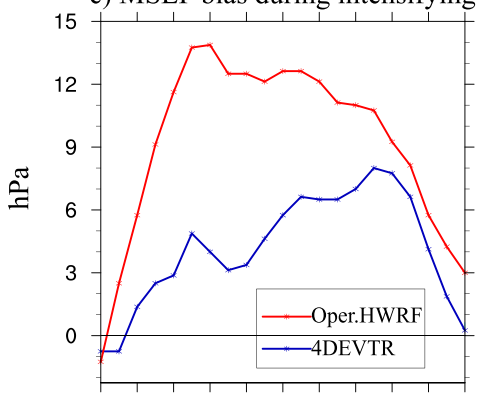

$001224 \quad 36 \quad 486072 \quad 8496108120$

Forecast Hour

e) MSLP @ 2014091400 UTC

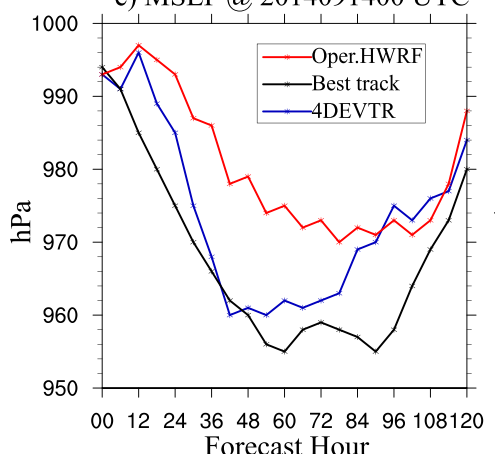

Forecast Hour

g) MSLP@2014091406 UTC

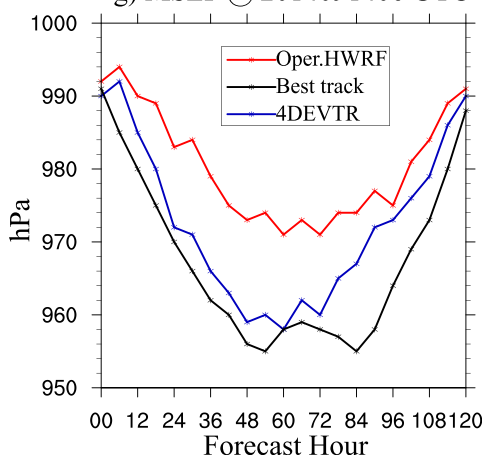

b) Vmax forecasts for all

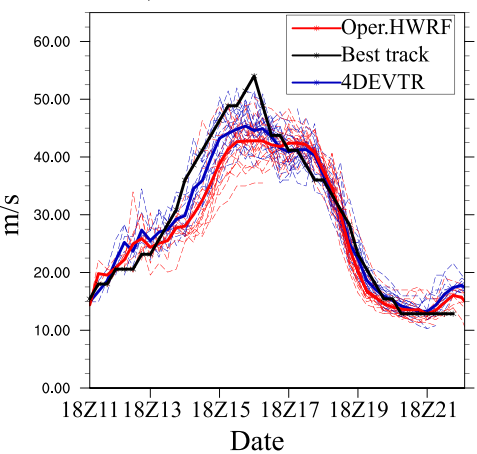

d) Vmax bias during intensifying

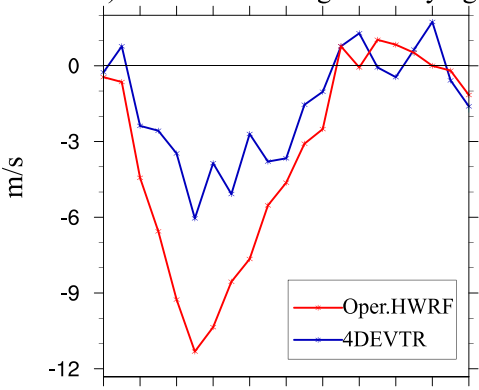

001224364860728496108120 Forecast Hour

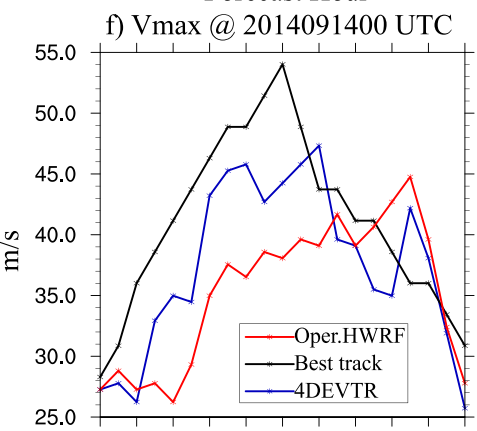

$0012243648 \quad 6072 \quad 8496108120$ Forecast Hour

h) Vmax @ 2014091406 UTC

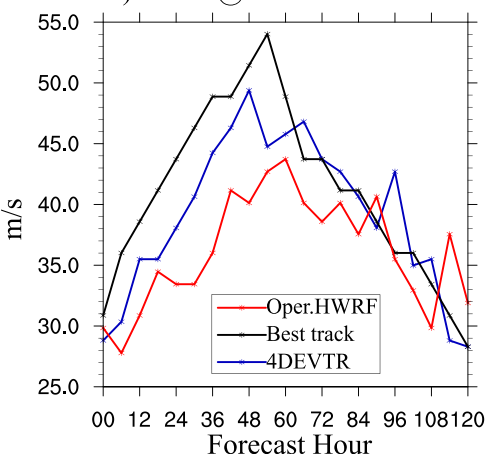

FIG. 9. (a) MSLP and (b) Vmax forecasts for all missions during Edouard for the operational HWRF (red), 4DEVTR (blue), and best track (black). The dashed lines denote the forecasts initialized from each DA cycle and the solid lines denote the multiforecast average valid at the same time. The (c) MSLP and (d) Vmax forecast error bias during the intensifying period [ $\operatorname{Vmax}$ change $>20 \mathrm{kt}(24 \mathrm{~h})^{-1}$ ] between 1200 UTC 13 Sep and 0600 UTC 15 Sep 2014 are given. The (e),(g) MSLP and (f),(h) Vmax forecast for (e),(f) 10th cycle at 0000 UTC and (g),(h) 11th cycle at 0600 UTC 14 Sep 2014 are also given. 
a) $4 \mathrm{DEVTR} 00 \mathrm{~h}$

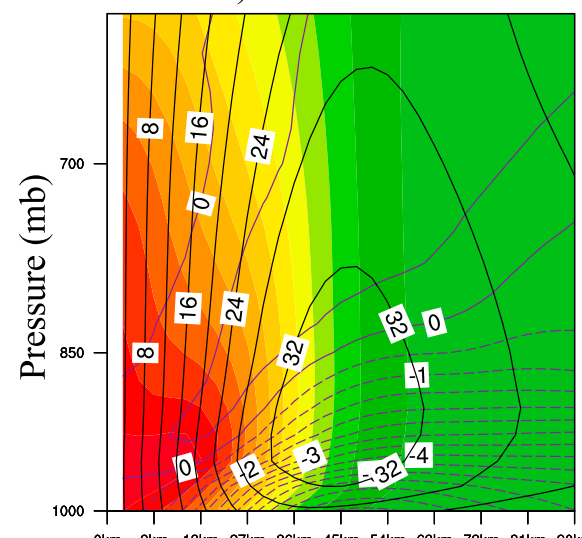

d) Oper.HWRF 00h

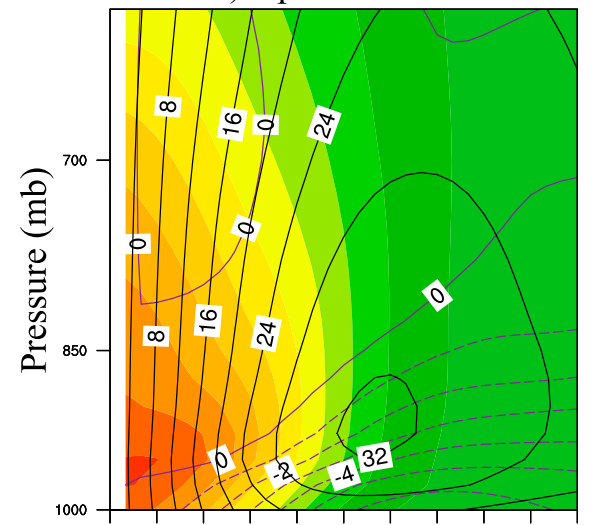

$0 \mathrm{~km} \quad 9 \mathrm{~km} \quad 18 \mathrm{~km} 27 \mathrm{~km} 36 \mathrm{~km} 45 \mathrm{~km} 54 \mathrm{~km} 63 \mathrm{~km} 72 \mathrm{~km} 81 \mathrm{~km} 90 \mathrm{~km}$

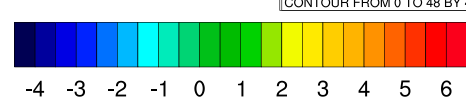

b) 4DEVTR 06h

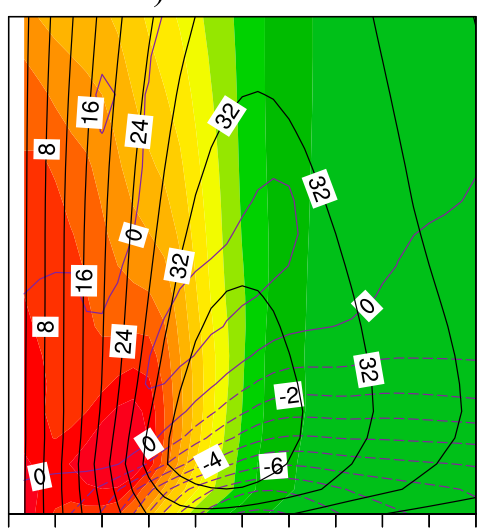

e) Oper.HWRF 06h

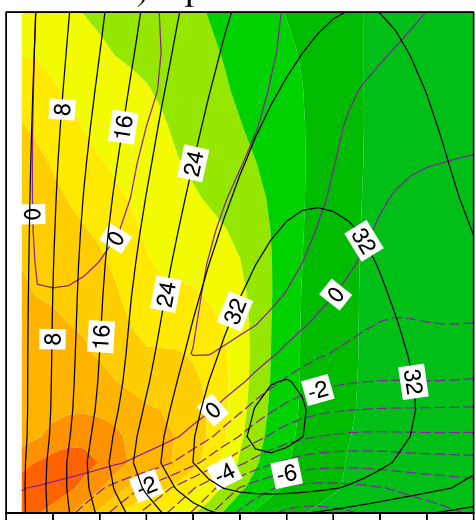

CONTOUR

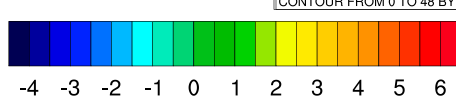

c) $4 \mathrm{DEVTR} 12 \mathrm{~h}$

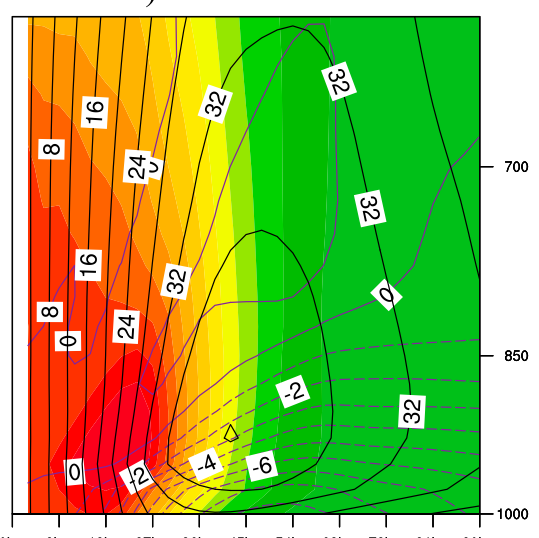

f) Oper.HWRF $12 \mathrm{~h}$

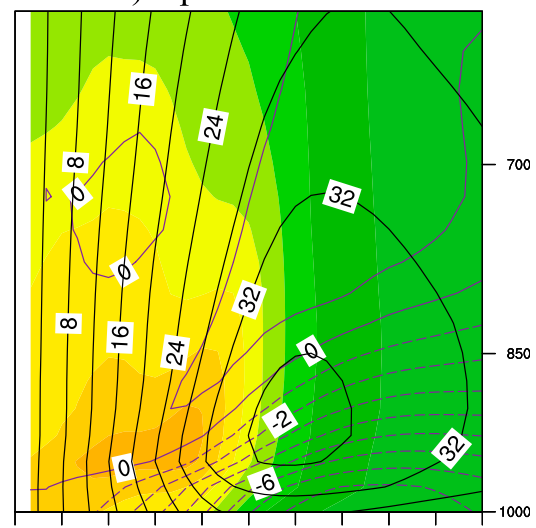

$44 \mathrm{~km} 63 \mathrm{~km} 72 \mathrm{~km} 81 \mathrm{~km} 90 \mathrm{~km}$ CONTOUR FROM 0 TO 48 BY 4

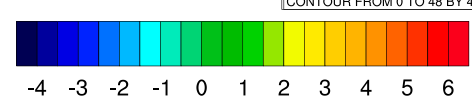

FIG. 10. Mean azimuth mean inertial stability (shading; $10^{-6} \mathrm{~s}^{-2}$ ), radial wind (purple contours; dashed for inflow, solid for outflow; $\mathrm{m} \mathrm{s}^{-1}$ ), and tangential wind (black contours; $\mathrm{m} \mathrm{s}^{-1}$ ) averaged over intensifying period (8 cycles in total from 1200 UTC 13 Sep to 0600 UTC 15 Sep 2014) for (a)-(c) 4DEVTR and (d)-(f) the operational HWRF and valid at (a),(d) 0; (b),(e) 6; and (c),(f) $12 \mathrm{~h}$.

MSLP and Vmax forecasts measured in terms of absolute errors are improved, although the improvements do not last long. The track, RMW, MSLP, and Vmax forecasts in 3DEV do not in general show consistent improvement over 3DEV-NEVR. However, systematic MSLP and Vmax error bias evolutions are in general better in 3DEV than in 3DEV-NEVR especially at the early lead times.

By design, 6-hourly hybrid 3DEnVar is not able to represent the fast-evolving error structures due to the lack of temporal flow dependency in the background error covariance. This issue is illustrated when Edouard went through rapid eyewall replacement, and the innercore TDR data were brief and unevenly distributed. Experiment 4DEVTR is designed to investigate the impact of using 4DEnVar DA in comparison with 3DEnVar for such situations. 4DEnVar improves the analyzed TC structure, as illustrated by its reduction of a spuriously strong wind maximum produced by 3DEnVar. Consistently, MSLP and Vmax forecasts are improved at early lead times initialized by 4DEnVar as compared with 3DEnVar, although slight degradations are found in the track forecasts. These track degradations warrant further investigations with a larger sample in the future.

The newly developed EnVar DA system is found to improve the MSLP and Vmax forecasts of Edouard (2014) due to the better analyses during the intensifying period compared to the operational HWRF. Further diagnostics indicate that the analyzed inner-core structure by EnVar possesses stronger inner-core inertial stability, a smaller radius of maximum wind, stronger tangential wind, and more symmetric inner-core vorticity structures during the intensifying period compared 
TABLE 3. Percentage of absolute vorticity variance explained by each wavenumber averaged over $1.5 \mathrm{RMW}$ radius during the intensifying period from 1200 UTC 13 Sep to 0600 UTC 15 Sep 2014.

\begin{tabular}{lcccccc}
\hline \hline Wavenumber & 0 & 1 & 2 & 3 & 4 & $>4$ \\
\hline 4DEVTR & 83.8457 & 8.73058 & 3.29733 & 1.53631 & 0.971752 & 1.61828 \\
Operational & 81.3286 & 8.7944 & 4.28238 & 1.91919 & 1.27799 & 2.3974 \\
HWRF & & & & & & \\
\hline
\end{tabular}

to the analysis produced by the operational HWRF system. This difference in structure may be responsible for the intensity forecast improvement.

The current study represents the first step of testing, evaluating, and understanding the newly developed hybrid DA system for HWRF. This system has served as a basis for the 2017 operational HWRF implementation and systematic experiments have been conducted before the implementation (J. Sippel 2017, personal communication). Although 32 cycles of continuously cycled DA and forecasts are performed in this study, the sample size is still relatively small. Further experiments are needed to systematically test the system with many more cases and with other types of inner-core observations. Additionally, more frequent assimilation cycles (e.g., using an hourly DA interval) offers an alternative way to take into account the quickly evolving error covariance. Therefore, further experiments are warranted to explore optimal DA configurations.

Acknowledgments. The research documented in this paper is supported by Grants NA12NWS4680012, NA14NWS4830008, NA14NWS4680021, and NA16NWS4680028 from the National Oceanic and Atmospheric Administration (NOAA) Hurricane Forecast Improvement Project (HFIP) and the ONR Grants N00014-14-1-0125 and N000141712111. The experiments are performed on the NOAA supercomputer Jet. Some results and descriptions were included in the abstract of the authors' AMS conference presentation, the abstract of the first author's dissertation seminar, and progress reports to the funding agencies. We greatly appreciate detailed and constructive comments from Jason Sippel and another reviewer of the paper.

\section{REFERENCES}

Abarca, S. F., M. T. Montgomery, S. A. Braun, and J. Dunion, 2016: On the secondary eyewall formation of Hurricane Edouard (2014). Mon. Wea. Rev., 144, 3321-3331, https://doi.org/10.1175/ MWR-D-15-0421.1.

Aksoy, A., S. Lorsolo, T. Vukicevic, K. J. Sellwood, S. D. Aberson, and F. Zhang, 2012: The HWRF Hurricane Ensemble Data Assimilation System (HEDAS) for high-resolution data: The impact of airborne Doppler radar observations in an OSSE. Mon. Wea. Rev., 140, 1843-1862, https://doi.org/10.1175/MWR-D-11-00212.1.

- S. D. Aberson, T. Vukicevic, K. J. Sellwood, S. Lorsolo, and X. Zhang, 2013: Assimilation of high-resolution tropical cyclone observations with an ensemble Kalman filter using NOAA/AOML/HRD's HEDAS: Evaluation of the 2008-11 vortex-scale analyses. Mon. Wea. Rev., 141, 1842-1865, https:// doi.org/10.1175/MWR-D-12-00194.1.

Bauer, P., P. Lopez, A. Benedetti, D. Salmond, and E. Moreau, 2006: Implementation of $1 \mathrm{D}+4 \mathrm{D}-\mathrm{Var}$ assimilation of precipitationaffected microwave radiances at ECMWF. I: 1D-Var. Quart. J. Roy. Meteor. Soc., 132, 2277-2306, https://doi.org/10.1256/ qj.05.189.

— similation at operational NWP centres. Quart. J. Roy. Meteor. Soc., 137, 1934-1951, https://doi.org/10.1002/qj.905.

Bender, M. A., R. J. Ross, R. E. Tuleya, and Y. Kurihara, 1993: Improvements in tropical cyclone track and intensity forecasts using the GFDL initialization system. Mon. Wea. Rev., 121, 2046-2061, https:// doi.org/10.1175/1520-0493(1993)121<2046:IITCTA>2.0.CO;2.

Chen, H., D. L. Zhang, J. Carton, and R. Atlas, 2011: On the rapid intensification of Hurricane Wilma (2005). Part I: Model prediction and structural changes. Wea. Forecasting, 26, 885-901, https://doi.org/10.1175/WAF-D-11-00001.1.

Chen, Y., and C. Snyder, 2007: Assimilating vortex position with an ensemble Kalman filter. Mon. Wea. Rev., 135, 1828-1845, https://doi.org/10.1175/MWR3351.1.

Coffey, J. J., G. A. Wick, R. E. Hood, J. P. Dunion, M. L. Black, and P. Kenul, 2015: Sensing hazards with operational unmanned technology: NOAA's application of the Global Hawk aircraft for high impact weather forecasting. 2015 Fall Meeting, San Francisco, CA, Amer. Geophys. Union, Abstract A53A-0362.

Davis, C., and L. F. Bosart, 2002: Numerical simulations of the genesis of Hurricane Diana (1984). Part II: Sensitivity of track and intensity prediction. Mon. Wea. Rev., 130, 1100-1124, https:// doi.org/10.1175/1520-0493(2002)130<1100:NSOTGO > 2.0.CO;2.

_ - W. Wang, J. Dudhia, and R. Torn, 2010: Does increased horizontal resolution improve hurricane wind forecasts? Wea. Forecasting, 25, 1826-1841, https://doi.org/10.1175/2010WAF2222423.1.

Doyle, J., and Coauthors, 2017: A view of tropical cyclones from above: The Tropical Cyclone Intensity experiment. Bull. Amer. Meteor. Soc., 98, 2113-2134, https://doi.org/10.1175/BAMS-D-16-0055.1.

Fels, S. B., and M. D. Schwarzkopf, 1975: The simplified exchange approximation: A new method for radiative transfer calculations. J. Atmos. Sci., 32, 1475-1488, https://doi.org/10.1175/ 1520-0469(1975)032<1475:TSEAAN >2.0.CO;2.

Ferrier, B. S., 2005: An efficient mixed-phase cloud and precipitation scheme for use in operational NWP models. Eos, Trans. Amer. Geophys. Union, 86 (Spring Meeting Suppl.), Abstract A42A-02.

Gamache, J. F., 2005: Real-time dissemination of hurricane wind fields determined from airborne Doppler radar data. Final Rep. on JHT Project, NOAA, 38 pp., http://www.nhc.noaa.gov/ jht/2003-2005reports/DOPLRgamache_JHTfinalreport.pdf.

Hack, J. J., and W. H. Schubert, 1986: Nonlinear response of atmospheric vortices to heating by organized cumulus convection. J. Atmos. Sci., 43, 1559-1573, https://doi.org/10.1175/ 1520-0469(1986)043<1559:NROAVT>2.0.CO;2.

Han, J., and H. L. Pan, 2011: Revision of convection and vertical diffusion schemes in the NCEP Global Forecast System. Wea. Forecasting, 26, 520-533, https://doi.org/10.1175/WAF-D-10-05038.1.

Holland, G., and R. Merrill, 1984: On the dynamics of tropical cyclone structural changes. Quart. J. Roy. Meteor. Soc., 110, 723-745, https://doi.org/10.1002/qj.49711046510. 
Hong, S. Y., and H. L. Pan, 1996: Nonlocal boundary layer vertical diffusion in a medium-range forecast model. Mon. Wea. Rev., 124, 2322-2339, https://doi.org/10.1175/1520-0493(1996)124<2322: NBLVDI $>2.0 . \mathrm{CO} ; 2$

Houze, R. A., S. S. Chen, B. F. Smull, W. C. Lee, and M. M. Bell, 2007: Hurricane intensity and eyewall replacement. Science, 315, 1235-1239, https://doi.org/10.1126/science.1135650.

Kleist, D. T., and K. Ide, 2015a: An OSSE-based evaluation of hybrid variational-ensemble data sssimilation for the NCEP GFS. Part I: System description and 3D-hybrid results. Mon. Wea. Rev., 143, 433-451, https://doi.org/10.1175/MWR-D-13-00351.1.

— , and — 2015b: An OSSE-based evaluation of hybrid variational-ensemble data assimilation for the NCEP GFS Part II: 4DEnVar and hybrid variants. Mon. Wea. Rev., 143, 452-470, https://doi.org/10.1175/MWR-D-13-00350.1.

Kurihara, Y., M. A. Bender, R. E. Tuleya, and R. J. Ross, 1990: Prediction experiments of Hurricane Gloria (1985) using a multiply nested movable mesh model. Mon. Wea. Rev., 118 2185-2198, https://doi.org/10.1175/1520-0493(1990)118<2185: PEOHGU $>2.0 . \mathrm{CO} ; 2$.

$\longrightarrow,-$ _, and R. J. Ross, 1993: An initialization scheme of hurricane models by vortex specification. Mon. Wea. Rev., 121, 2030-2045, https://doi.org/10.1175/1520-0493(1993)121<2030: AISOHM $>2.0 . \mathrm{CO} ; 2$.

,,-- R. E. Tuleya, and R. J. Ross, 1995: Improvements in the GFDL hurricane prediction system. Mon. Wea. Rev., 123 2791-2801, https://doi.org/10.1175/1520-0493(1995)123<2791: IITGHP $>2.0 . \mathrm{CO} ; 2$.

Lacis, A. A., and J. Hansen, 1974: A parameterization for the absorption of solar radiation in the earth's atmosphere. J. Atmos. Sci., 31, 118-133, https://doi.org/10.1175/1520-0469(1974)031<0118: APFTAO $>2.0 . \mathrm{CO} ; 2$.

Li, X., J. Ming, M. Xue, Y. Wang, and K. Zhao, 2015: Implementation of a dynamic equation constraint based on the steady state momentum equations within the WRF hybrid ensemble-3DVar data assimilation system and test with radar T-TREC wind assimilation for Tropical Cyclone Chanthu (2010). J. Geophys. Res. Atmos., 120, 4017-4039, https://doi.org/10.1002/2014JD022706.

Li, Y., 2015: Assimilation of radar observations with ensemble variational hybrid data assimilation method for the initialization and prediction of hurricanes. Ph.D. dissertation, University of Oklahoma, 124 pp., http://hdl.handle.net/11244/14050.

— X. Wang, and M. Xue, 2012: Assimilation of radar radial velocity data with the WRF hybrid ensemble-3DVAR system for the prediction of Hurricane Ike (2008). Mon. Wea. Rev., 140, 3507-3524, https://doi.org/10.1175/MWR-D-12-00043.1.

Liu, Q., T. Marchok, H. L. Pan, M. Bender, and S. J. Lord, 2000: Improvements in hurricane initialization and forecasting at NCEP with global and regional (GFDL) models. NOAA Tech. Procedures Bull. 472, NOAA, 7 pp., http://www.nws.noaa.gov/ om/tpb/472body.htm.

- S. Lord, N. Surgi, Y. Zhu, R. Wobus, Z. Toth, and T. Marchok, 2006: Hurricane relocation in Global Ensemble Forecast System. 27th Conf. on Hurricanes and Tropical Meteorology, Monterey, CA, Amer. Meteor. Soc., P5.13, https://ams.confex.com/ams/ pdfpapers/108503.pdf.

Lord, S. J., 1991: A bogusing system for vortex circulations in the National Meteorological Center global forecast model. Preprints, 19th Conf. on Hurricanes and Tropical Meteorology, Miami, FL, Amer. Meteor. Soc., 328-330.

Lu, X., X. Wang, Y. Li, M. Tong, and X. Ma, 2017: GSI-based ensemble-variational hybrid data assimilation for HWRF for hurricane initialization and prediction: Impact of various error covariances for airborne radar observation assimilation. Quart. J. Roy. Meteor. Soc., 143, 223-239, https://doi.org/10.1002/qj.2914.

Nolan, D. S., and M. T. Montgomery, 2002: Nonhydrostatic, three-dimensional perturbations to balanced, hurricane-like vortices. Part I: Linearized formulation, stability, and evolution. J. Atmos. Sci., 59, 2989-3020, https://doi.org/10.1175/ 1520-0469(2002)059<2989:NTDPTB > 2.0.CO;2.

, and L. D. Grasso, 2003: Nonhydrostatic, three-dimensional perturbations to balanced, hurricane-like vortices. Part II: Symmetric response and nonlinear simulations. J. Atmos. Sci., 60, 2717-2745, https://doi.org/10.1175/1520-0469(2003)060<2717:NTPTBH > 2.0.CO;2. , Y. Moon, and D. P. Stern, 2007: Tropical cyclone intensification from asymmetric convection: Energetics and efficiency. J. Atmos. Sci., 64, 3377-3405, https://doi.org/10.1175/ JAS3988.1.

Poterjoy, J., and F. Zhang, 2014: Intercomparison and coupling of ensemble and four-dimensional variational data assimilation methods for the analysis and forecasting of Hurricane Karl (2010). Mon. Wea. Rev., 142, 3347-3364, https://doi.org/ 10.1175/MWR-D-13-00394.1.

and Y. Weng, 2014: The effects of sampling errors on the EnKF assimilation of inner-core hurricane observations. Mon. Wea. Rev., 142, 1609-1630, https://doi.org/10.1175/MWR-D-13-00305.1.

$\mathrm{Pu}, \mathrm{Z}$., and S. A. Braun, 2001: Evaluation of bogus vortex techniques with four-dimensional variational data assimilation. Mon. Wea. Rev., 129, 2023-2039, https://doi.org/10.1175/ 1520-0493(2001)129<2023:EOBVTW>2.0.CO;2.

Reasor, P. D., M. T. Montgomery, F. D. Marks, and J. F. Gamache, 2000: Low-wavenumber structure and evolution of the hurricane inner core observed by airborne dual-Doppler radar. Mon. Wea. Rev., 128, 1653-1680, https://doi.org/10.1175/1520-0493(2000)128<1653: LWSAEO $>2.0 . \mathrm{CO} ; 2$.

Rogers, R., P. Reasor, and S. Lorsolo, 2013a: Airborne Doppler observations of the inner-core structural differences between intensifying and steady-state tropical cyclones. Mon. Wea. Rev., 141, 2970-2991, https://doi.org/10.1175/MWR-D-12-00357.1.

_ , and Coauthors, 2013b: NOAA's Hurricane Intensity Forecasting Experiment: A progress report. Bull. Amer. Meteor. Soc., 94, 859-882, https://doi.org/10.1175/BAMS-D-12-00089.1.

Schubert, W. H., and J. J. Hack, 1982: Inertial stability and tropical cyclone development. J. Atmos. Sci., 39, 1687-1697, https://doi.org/ 10.1175/1520-0469(1982)039<1687:ISATCD > 2.0.CO;2.

Schwartz, C. S., 2016: Improving large-domain convection-allowing forecasts with high-resolution analyses and ensemble data assimilation. Mon. Wea. Rev., 144, 1777-1803, https://doi.org/ 10.1175/MWR-D-15-0286.1.

— Z. Liu, X. Y. Huang, Y. H. Kuo, and C. T. Fong, 2013: Comparing limited-area 3DVAR and hybrid variational-ensemble data assimilation methods for typhoon track forecasts: Sensitivity to outer loops and vortex relocation. Mon. Wea. Rev., 141, 4350-4372, https://doi.org/10.1175/MWR-D-13-00028.1.

- — $\longrightarrow$, and —, 2015: Sensitivity of limited-area hybrid variational-ensemble analyses and forecasts to ensemble perturbation resolution. Mon. Wea. Rev., 143, 3454-3477, https://doi.org/10.1175/MWR-D-14-00259.1.

Schwarzkopf, M. D., and S. B. Fels, 1991: The simplified exchange method revisited: An accurate, rapid method for computation of infrared cooling rates and fluxes. J. Geophys. Res., 96, 9075-9096, https://doi.org/10.1029/89JD01598.

Stewart, S. R., 2014: National Hurricane Center Tropical Cyclone Report: Hurricane Edouard (11-19 September 2014). NHC Rep. AL062014, 19 pp., http://www.nhc.noaa.gov/data/tcr/ AL062014_Edouard.pdf. 
Tallapragada, V., and Coauthors, 2014: Hurricane Weather Research and Forecasting (HWRF) Model: 2014 scientific documentation. HWRF v3.6a, NCAR Developmental Testbed Center Rep., 105 pp., http://www.dtcenter.org/HurrWRF/users/ docs/scientific_documents/HWRFv3.6a_ScientificDoc.pdf.

Thu, T. V., and T. N. Krishnamurti, 1992: Vortex initialization for typhoon track prediction. Meteor. Atmos. Phys., 47, 117-126, https://doi.org/10.1007/BF01025612.

Tong, M., V. Tallapragada, E. Liu, W. Wang, C. Kieu, Q. Liu, and B. Zhang, 2014: Impact of assimilating aircraft reconnaissance observations in operational HWRF. HFIP Annual Review Meeting, College Park, MD, HFIP, 21 pp., http://www.hfip.org/events/annual_ meeting_nov_2014/wed/15_Tong_2014_HFIP_annual_meeting.pdf.

Torn, R. D., 2010: Performance of a mesoscale ensemble Kalman filter (EnKF) during the NOAA High-Resolution Hurricane test. Mon. Wea. Rev., 138, 4375-4392, https://doi.org/10.1175/ 2010MWR3361.1.

—_, and G. J. Hakim, 2009: Ensemble data assimilation applied to RAINEX observations of Hurricane Katrina (2005). Mon. Wea. Rev., 137, 2817-2829, https://doi.org/10.1175/2009MWR2656.1.

Trahan, S., and L. Sparling, 2012: An analysis of NCEP Tropical Cyclone Vitals and potential effects on forecasting models. Wea. Forecasting, 27, 744-756, https://doi.org/10.1175/WAF-D-11-00063.1.

Tuleya, R. E., 1994: Tropical storm development and decay: Sensitivity to surface boundary conditions. Mon. Wea. Rev., 122, 291-304, https://doi.org/10.1175/1520-0493(1994)122<0291: TSDADS $>2.0 . \mathrm{CO} ; 2$.

Wang, X., 2010: Incorporating ensemble covariance in the gridpoint statistical interpolation variational minimization: A mathematical framework. Mon. Wea. Rev., 138, 2990-2995, https://doi.org/10.1175/2010MWR3245.1.

—, 2011: Application of the WRF hybrid ETKF-3DVAR data assimilation system for hurricane track forecasts. Wea. Forecasting, 26, 868-884, https://doi.org/10.1175/WAF-D-10-05058.1.

, and T. Lei, 2014: GSI-based four dimensional ensemblevariational (4DEnsVar) data assimilation: Formulation and single resolution experiments with real data for NCEP Global Forecast System. Mon. Wea. Rev., 142, 3303-3325, https://doi.org/ 10.1175/MWR-D-13-00303.1.

—, D. Barker, C. Snyder, and T. M. Hamill, 2008a: A hybrid ETKF-3DVAR data assimilation scheme for the WRF model. Part I: Observing system simulation experiment. Mon. Wea. Rev., 136, 5116-5131, https://doi.org/10.1175/2008MWR2444.1.

,,,--- and,$- 2008 \mathrm{~b}$ : A hybrid ETKF-3DVAR data assimilation scheme for the WRF model. Part II: Real observation experiments. Mon. Wea. Rev., 136, 5132-5147, https:// doi.org/10.1175/2008MWR2445.1.

—, D. Parrish, D. Kleist, and J. Whitaker, 2013: GSI 3DVarbased ensemble-variational hybrid data assimilation for NCEP Global Forecast System: Single-resolution experiments. Mon. Wea. Rev., 141, 4098-4117, https://doi.org/ 10.1175/MWR-D-12-00141.1.

Weng, Y., and F. Zhang, 2012: Assimilating airborne Doppler radar observations with an ensemble Kalman filter for convection-permitting hurricane initialization and prediction: Katrina (2005). Mon. Wea. Rev., 140, 841-859, https://doi.org/ 10.1175/2011MWR3602.1.
Whitaker, J. S., and T. M. Hamill, 2002: Ensemble data assimilation without perturbed observations. Mon. Wea. Rev., 130, 1913-1924, https://doi.org/10.1175/1520-0493(2002)130<1913: EDAWPO $>2.0 . \mathrm{CO} ; 2$.

— errors in ensemble data assimilation. Mon. Wea. Rev., 140, 3078-3089, https://doi.org/10.1175/MWR-D-11-00276.1.

Xiao, Q., X. Zhang, C. Davis, J. Tuttle, G. Holland, and P. J. Fitzpatrick, 2009: Experiments of hurricane initialization with airborne Doppler radar data for the Advanced Research Hurricane WRF (AHW) model. Mon. Wea. Rev., 137, 2758-2777, https://doi.org/10.1175/2009MWR2828.1.

Xue, M., J. Schleif, F. Kong, K. W. Thomas, Y. Wang, and K. Zhu, 2013: Track and intensity forecasting of hurricanes: Impact of convection-permitting resolution and global ensemble Kalman filter analysis on 2010 Atlantic season forecasts. Wea. Forecasting, 28, 1366-1384, https://doi.org/10.1175/WAF-D-12-00063.1.

Yang, C., Z. Liu, J. Bresch, S. R. H. Rizvi, X. Y. Huang, and J. Min, 2016: AMSR2 all-sky radiance assimilation and its impact on the analysis and forecast of Hurricane Sandy with a limited-area data assimilation system. Tellus, 68A, 30917, https://doi.org/ 10.3402/tellusa.v68.30917.

Yang, S.-C., K.-J. Lin, T. Miyoshi, and E. Kalnay, 2013: Improving the spin-up of regional EnKF for typhoon assimilation and forecasting with Typhoon Sinlaku (2008). Tellus, 65A, 20804, https://doi.org/10.3402/tellusa.v65i0.20804.

Zhang, F., and Y. Weng, 2015: Predicting hurricane intensity and associated hazards: A five-year real-time forecast experiment with assimilation of airborne Doppler radar observations. Bull. Amer. Meteor. Soc., 96, 25-33, https://doi.org/10.1175/BAMS-D-13-00231.1.

,-- J J. A. Sippel, Z. Meng, and C. H. Bishop, 2009: Cloudresolving hurricane initialization and prediction through assimilation of Doppler radar observations with an ensemble Kalman filter. Mon. Wea. Rev., 137, 2105-2125, https://doi.org/ 10.1175/2009MWR2645.1.

_,- _ J J. F. Gamache, and F. D. Marks, 2011: Performance of convection-permitting hurricane initialization and prediction during 2008-2010 with ensemble data assimilation of inner-core airborne Doppler radar observations. Geophys. Res. Lett., 38, L15810, https://doi.org/10.1029/ 2011GL048469.

—, M. Minamide, and E. E. Clothiaux, 2016: Potential impacts of assimilating all-sky infrared satellite radiances from GOES-R on convection-permitting analysis and prediction of tropical cyclones. Geophys. Res. Lett., 43, 2954-2963, https://doi.org/ 10.1002/2016GL068468.

Zhao, C., H. Shao, and L. Bernardet, 2015: Applications of the GSI-hybrid data assimilation for high-resolution tropical storm forecasts: Tackling the intensity spindown issue in 2014 HWRF. 16th WRF Users' Workshop, Boulder, CO, NCAR, http://www.dtcenter.org/eval/ data_assim/publications/GSI-Hybrid \%20at \% 202015\% 20WRFUsersWorkshop.v2_poster.pdf.

Zhu, T., D. L. Zhang, and F. H. Weng, 2004: Numerical simulation of Hurricane Bonnie (1998). Part I: Eyewall evolution and intensity changes. Mon. Wea. Rev., 132, 225-241, https://doi.org/ 10.1175/1520-0493(2004)132<0225:NSOHBP > 2.0.CO;2. 